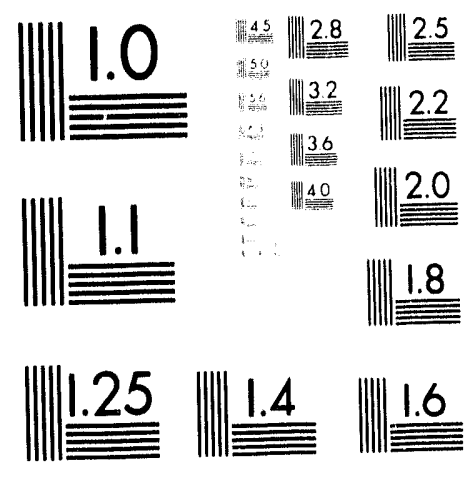



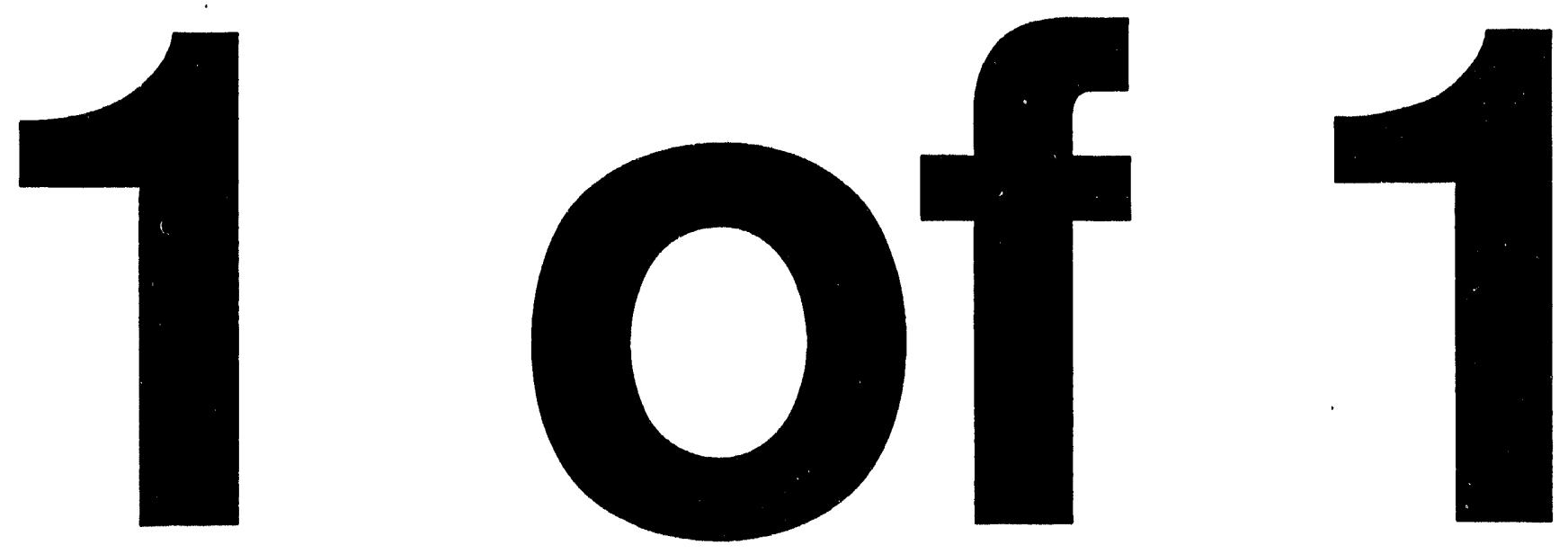
LBL-32505

\title{
A Two-Dimensional Dispersion Module for the TOUGH2 Simulator
}

\author{
Curtis M. Oldenburg and Karsten Pruess \\ Earth Sciences Division \\ Lawrence Berkeley Laboratory \\ University of California \\ Berkeley, California 94720
}

September 1993

This work was supported by the WIPP project, Sandia National Laboratories, under Document No. 129847, and by the Assistant Secretary for Energy Efficiency and Renewable Energy, Geothermal Division, of the U.S. Department of Energy, through Contract No. DE-AC03-76SF00098. 


\begin{abstract}
A standard model for hydrodynamic dispersion has been added to TOUGH2. The dispersion model, intended for use with the EOS7 fluid properties module, accounts for the effects of hydrodynamic dispersion and molecular diffusion in two-dimensional rectangular domains. Because the model requires Darcy velocity and species concentration gradient vectors at all connections, known vector components (perpendicular to the grid block interfaces) from neighboring connections are interpolated to form the unknown components (parallel to the grid block interfaces) at each connection. Thus the dispersive fluxes depend not only on the primary variables of the two connected grid blocks but on all primary variables of the six neighbor grid blocks of each interface. This gives rise to added terms in the Jacobian matrix relative to standard TOUGH2 where fluxes depend only on primary variables in the two connected grid blocks. For flexibility in implementing boundary conditions, the model allows the user to define a flow domain that is a subset of the calculation domain. The PARAM and SELEC blocks of the TOUGH2 input file are used to specify parameters and boundary condition options for the dispersion module. The dispersion module has been verified by comparing computed results to analytical solutions. As an introduction to applications, we demonstrate the solution of a difficult twodimensional flow problem with variable salinity and strong coupling between dispersive and advective flow.
\end{abstract}




\section{Contents}

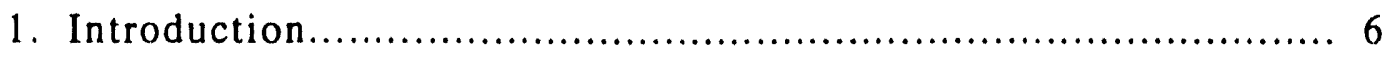

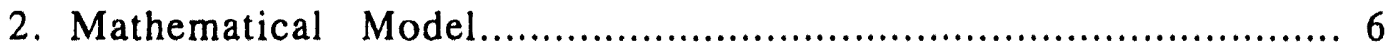

3. Implementation

3.1 Interpolation of Velocity and Gradient Vectors......................... 9

3.2 Boundary Conditions............................................... 16

3.3 Restrictions on the Mesh............................................ 19

3.4 Interface Conductivity............................................... 20

4. Using the dispersion module

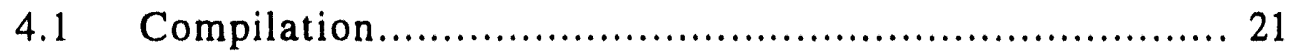

4.2 Input Formats...................................................... 23

5. Sample problems

5.1 One-Dimensional Transport with Dispersion.......................... 26

5.2 Two-Dimensional Transport with Dispersion.......................... 32

5.3 Two-Dimensional Transport with Variable Density...................... 41

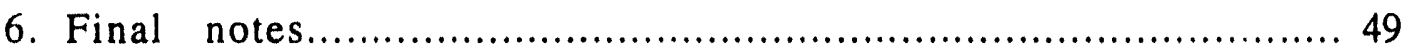

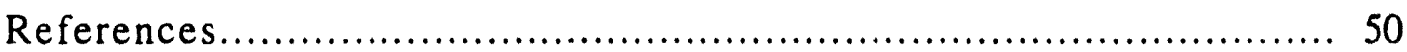

Acknowledgments......................................................... 52

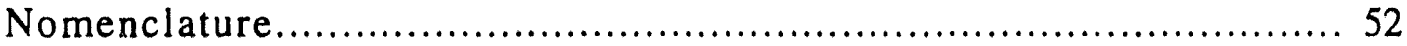

Appendix 1. A Diagnostic Test Problem................................................ 55 


\section{List of Figures}

Fig. 1. Single grid block with volume $V_{n}$ and surface area $\Gamma_{n} \ldots \ldots \ldots \ldots \ldots \ldots \ldots \ldots . . . . . . .7$

Fig. 2. Terminology for grid blocks and interfaces in the $\mathrm{Y}-\mathrm{Z}$ plane................ 11

Fig. 3. Interfacial distances relevant to the interpolation of vestors................... 12

Fig. 4. Velocity or concentration gradient vector components at interfaces......... 13

Fig. 5. The grid blocks involved in calculating dispersive flux....................... 15

Fig. 6. Sketch of a flow domain within a calculation domain........................ 16

Fig. 7. Interpolation of parallel vector component at flow boundary................ 18

Fig. 8. Interpolation of perpendicular vector component at flow boundary........ 18

Fig. 9. Domain for one-dimensional dispersion problem............................ 27

Fig. 10. Input file for the one-dimensional dispersion problem....................... 28

Fig. 11. Brine concentration for one-dimensional dispersion at $t=20$ days......... 29

Fig. 12. One-dimensional tracer transport results at $t=20$ days..................... 30

Fig. 13. Two-dimensional tracer transport problem................................... 32

Fig. 14. Coarse and fine discretizations for two-dimensional tracer transport....... 33

Fig. 15. Input file for the two-dimensional transport problem......................... 34

Fig. 16. Contour plot of tracer concentration at $t=20$ days......................... 37

Fig. 17. Tracer brine concentration profile A-A'.................................. 38

Fig. 18. Tracer brine concentration profile B-B'

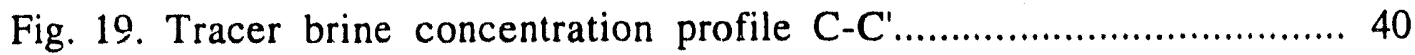

Fig. 20. Variable density brine flow and dispersion problem.......................... 42

Fig. 21. Input file for the variable density dispersion problem........................ 45

Fig. 22. Results for the variable density dispersion problem........................ 48

Fig. 23. Input file for the rdis1 test problem.......................................... 56

Fig. 24. Discretized domain for the rdis1 test problem............................... 58

Fig. 25. Printout from rdis 1 test problem.............................................. 59 


\section{List of Tables}

Table 1. Summary of program units in T2DM...................................... 22

Table 2. Molecular diffusivities for two phases and three components.............. 26

Table 3. Parameters for the variable density dispersion problem...................... 44 


\section{Introduction}

We have added a general model for Fickian solute dispersion to the multiphase porous media transport code TOUGH2. Used in conjunction with EOS7, an equation of state module for water, brine, and air, the TOUGH2 Dispersion Module (T2DM) models brine transport including the effects of hydrodynamic dispersion and molecular diffusion in rectangular, two-dimensional regions. Diffusion and hydrodynamic dispersion of vapor and air in the gas phase are also modeled. The dispersion module consists of a main program and eight subroutines which must be compiled and linked in front of the standard TOUGH2 Version 1.0. The dispersion module is entirely an extension of TOUGH2. As such, none of the modeling capabilities of TOUGH2 are lost with the use of T2DM, except for the current restriction to two dimensions and a rectangular mesh. This report presents a discussion of the dispersion model used and its implementation in TOUGH2, followed by instructions for using the new module. Example problems are solved to verify the model against available analytical and numerical solutions. This report on T2DM is an extension to existing TOUGH2 documentaition (Pruess, 1987, 1991).

\section{Mathematical Model}

The general conservation equations solved by the integral finite difference method (IFDM) in TOUGH2 consist of balances between mass accumulation and flux and source terms over all grid blocks $V_{n}$ into which the flow domain $V$ has been partitioned (see Fig. 1):

$$
\frac{\mathrm{d}}{\mathrm{dt}} \int_{V_{n}} M^{(\kappa)} \mathrm{d} V=\int_{\Gamma_{n}} \mathbf{F} \cdot \mathbf{n} \mathrm{d} \Gamma+\int_{V_{n}} q^{(\kappa)} \mathrm{d} V
$$




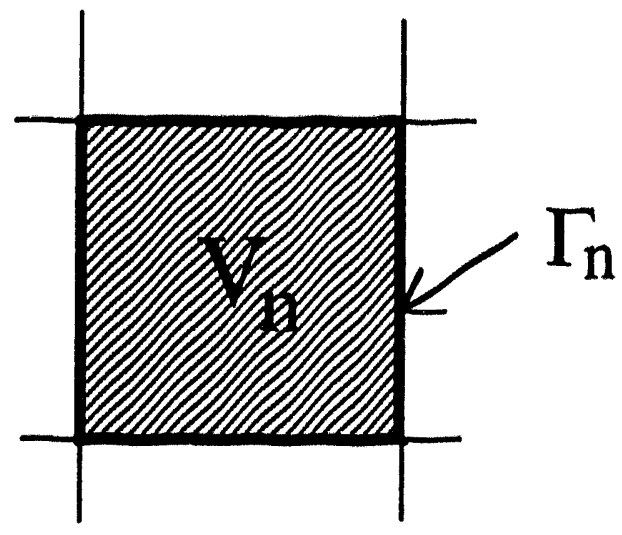

Fig. 1. Single grid block $n$ with volume $V_{n}$ and surface area $\Gamma_{n}$.

In Eq. 1, the index $n=1, \ldots, N$ corresponds to the grid blocks. The index $x=1, \ldots$, $\mathrm{NK}+1$ corresponds to the $\mathrm{NK}$ fluid components and heat. The $n$th two-dimensional grid block with volume $V_{n}$ and surface area $\Gamma_{n}$ is shown in Fig. 1. The mass accumulation term $(M)$ in Eq. 1 is given by

$$
M^{(\kappa)}=\phi \sum_{\beta=1}^{N P H} S_{\beta} \rho_{\beta} X_{\beta}^{(\kappa)}
$$

(see Nomenclature for definition of symbols). The flux term has contributions from both the phase flux and from dispersion and can be written

$$
F^{(\kappa)}=\sum_{\beta=1}^{N P H}\left(X_{\beta}^{(\kappa)} F_{\beta}-\rho_{\beta} \bar{D}_{\beta}^{(\kappa)} \nabla X_{\beta}^{(\kappa)}\right)
$$

The first term on the right-hand side of Eq. 3 is the phase flux term which accounts for the flux of component $\kappa$ arising from advection of the phases containing $\kappa$. Phase flux is written as a multiphase version of Darcy's Law: 


$$
\mathbf{F}_{\beta}=\rho_{\beta} \mathbf{u}_{\beta}=-k \frac{k_{r, \beta}}{\mu_{\beta}} \rho_{\beta}\left(\nabla P_{\beta}-\rho_{\beta} \mathbf{g}\right)
$$

The second term on the right-hand side of Eq. 3 is the dispersion term with the dispersion tensor indicated by the overbar. This report focuses on our implementation of a dispersion model which, for all phases $\beta=1, \ldots$, NPH present, accounts for the contribution to the flux of component $\kappa$ due to dispersion.

We have employed a general model which accounts for both molecular diffusion and hydrodynamic dispersion (deMarsily, 1986). The bold $\overline{\mathbf{D}}$ in Eq. 3 is the dispersion tensor, a second order, symmetric tensor with one principal direction in the average (Darcy) flow direction, and the other normal to it. The dispersion model is written in terms of dispersion coefficients in the longitudinal $\left(D_{L}\right)$ and transverse $\left(D_{T}\right)$ directions relative to the flow direction where

$$
\begin{aligned}
& D_{L, \beta}^{\kappa}=\phi \cdot S_{\beta} \cdot \tau \cdot d_{\beta}^{\kappa}+\alpha_{L} u_{\beta} \\
& D_{T, \beta}^{\kappa}=\phi \cdot S_{\beta} \cdot \tau \cdot d_{\beta}^{\kappa}+\alpha_{T} u_{\beta}
\end{aligned}
$$

Here $\phi$ is the porosity, $S_{\beta}$ is the saturation of phase $\beta, \tau$ is the tortuosity of the medium, $d_{\beta}^{\kappa}$ is the molecular diffusivity for component $K$ in phase $\beta, \alpha_{L}$ is the intrinsic longitudinal dispersion coefficient (often called the longitudinal dispersivity, or longitudinal dispersion length), $\alpha_{r}$ is the intrinsic transverse dispersion coefficient, and $u_{\beta}$ is the magnitude of the Darcy velocity of phase $\beta$. We assume that the dispersivities are (a) properties of the porous medium, (b) the same for all phases and, (c) independent of phase saturation. This is an expedient at present as little is known about the dependence of dispersivities on wettability and saturation of different phases (Sahimi et al., 1986a, b). The dispersion tensor of Eq. 3 can be written as 


$$
\overline{\mathbf{D}}_{\beta}^{\kappa}=D_{T, \beta}^{\kappa} \overline{\mathbf{I}}+\frac{\left(D_{L, \beta}^{\kappa}-D_{T, \beta}^{\kappa}\right)}{u_{\beta}^{2}} \mathbf{u}_{\beta} \mathbf{u}_{\beta}
$$

or, in terms of components relative to orthogonal coordinate axes $i$ and $j$,

$$
D_{i j, \beta}^{\kappa}=D_{T, \beta}^{x} \delta_{i j}+\frac{\left(D_{L, \beta}^{\kappa}-D_{T, \beta}^{\kappa}\right)}{u_{\beta}^{2}} u_{i} u_{j}
$$

where $\delta_{i j}$ is the Kronecker delta function.

Substituting Eq. 7 into Eq. 3 gives the mass flux of component $\boldsymbol{x}$ due to molecular diffusion and hydrodynamic dispersion in phase $\beta$ :

$\mathbf{F}_{\beta, d}{ }^{(\kappa)}=-\rho_{\beta} \overline{\mathbf{D}}_{\beta}^{(\kappa)} \nabla X_{\beta}^{(\kappa)}=-\rho_{\beta} D_{T, \beta}^{\kappa} \nabla X_{\beta}^{(\kappa)} \cdot \rho_{\beta} \frac{\left(D_{L, \beta}^{\kappa}-D_{T, \beta}^{\kappa}\right)}{u_{\beta}{ }^{2}} \mathbf{u}_{\beta}\left(\mathbf{u}_{\beta} \cdot \nabla X_{\beta}^{(\kappa)}\right)$

This term is calculated in the present dispersion module and is added to the phase flux (first term in Eq. 3). In TOUGH2, the discretized mass and energy balance equations are solved simultaneously taking all coupling terms into account. Because the disperson module simply augments the flux term by the flux due to dispersion, none of the many capabilities of the standard TOUGH2 are taken away with the use of T2DM.

\section{Implementation}

\subsection{Interpolation of Velocity and Gradient Vectors}

The above dispersion model has been implemented in TOUGH 2 with the addition of the new subroutine DISF which calculates the dispersive fluxes. Two other new subroutines (DOT, and OUTDF) have been added and minor modifications for interfacing with the dispersion routines have been made to the main program and to five subroutines. In the standard TOUGH 2 methodology, the geometric data for grid blocks and their flow 
connections are listed as scattered data with no reference to any global system of coordinates whatsoever. The dispersive fluxes depend on the vector quantities of Darcy velocity and species concentration gradient at each interface. In two-dimensional flow there are two vector components: the component perpendicular to the interface can be calculated directly from thermodynamic parameters of the two neighboring grid blocks; the component parallel to the interface must be interpolated from four direct components that involve thermodynamic parameters of six neighboring grid blocks. In order to perform this interpolation, some coordinate system must be referenced. Shown in Fig. 2 is the terminology of the local neighboring grid blocks, vertices, and interfaces used to calculate the required interface quantities. In this terminology, grid block nodes are referred to by lower-case letters while vertices and interfaces are referred to by upper-case letters. For example, using grid block $\mathrm{m}$ as the reference grid block, the lower case grid blocks e, $\mathrm{s}, \mathrm{w}$, and $\mathrm{n}$ are the grid blocks to the east, soutt, west, and north, respectively. The uppercase E, S, W, and $\mathrm{N}$ are the interfaces to the east, south, west and north. Vertices (intersections of lines forming boundaries of grid blocks) are given by the upper-case letter pairs corresponding to their direction away from $\mathrm{m}$.

In subroutine DISF, the Darcy velocity vector and the gradient vector of the mass fraction of component $\kappa$ are interpolated from the interface centers onto the vertices and then directly onto the center of the interface where they will be needed to calculate the dispersive flux by Eq. 9. For example, with reference to Fig. 3, the Y-component (U) of the Darcy velocity vector at interface $\mathrm{N}$ is given by

$$
U_{N}=\frac{U_{N W}+U_{N E}}{2}
$$

where

$$
U_{N W}=\frac{U_{n W} D_{1 N}+U_{W} D_{2 N}}{D_{1 N}+D_{2 N}}
$$



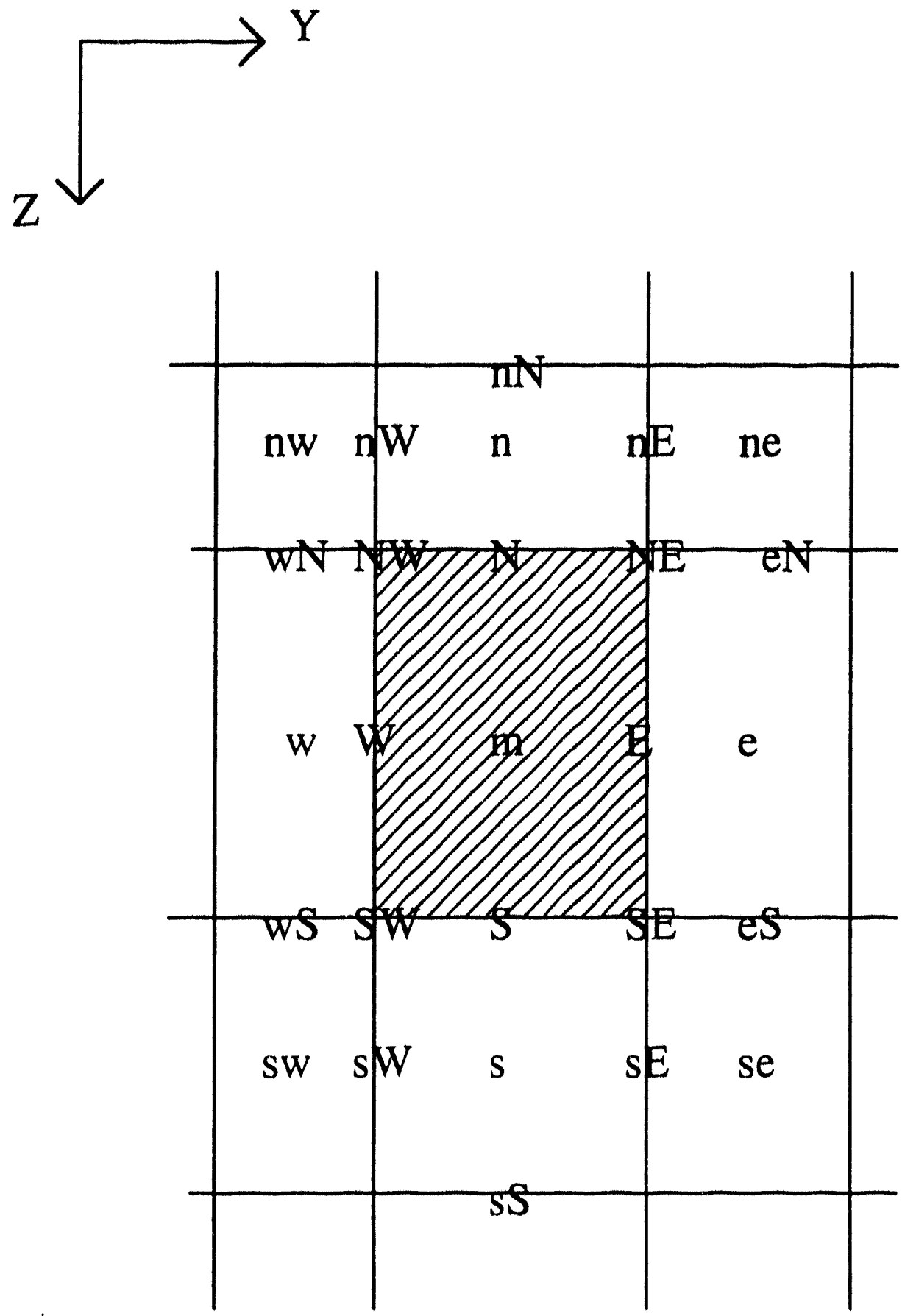

Fig. 2. Terminology for the grid blocks and interfaces in the neighborhood of grid block $m$ in the $\mathrm{Y}-\mathrm{Z}$ coordinate plane. Lower case letters refer to nodes, upper case letters to interfaces between grid blocks. 


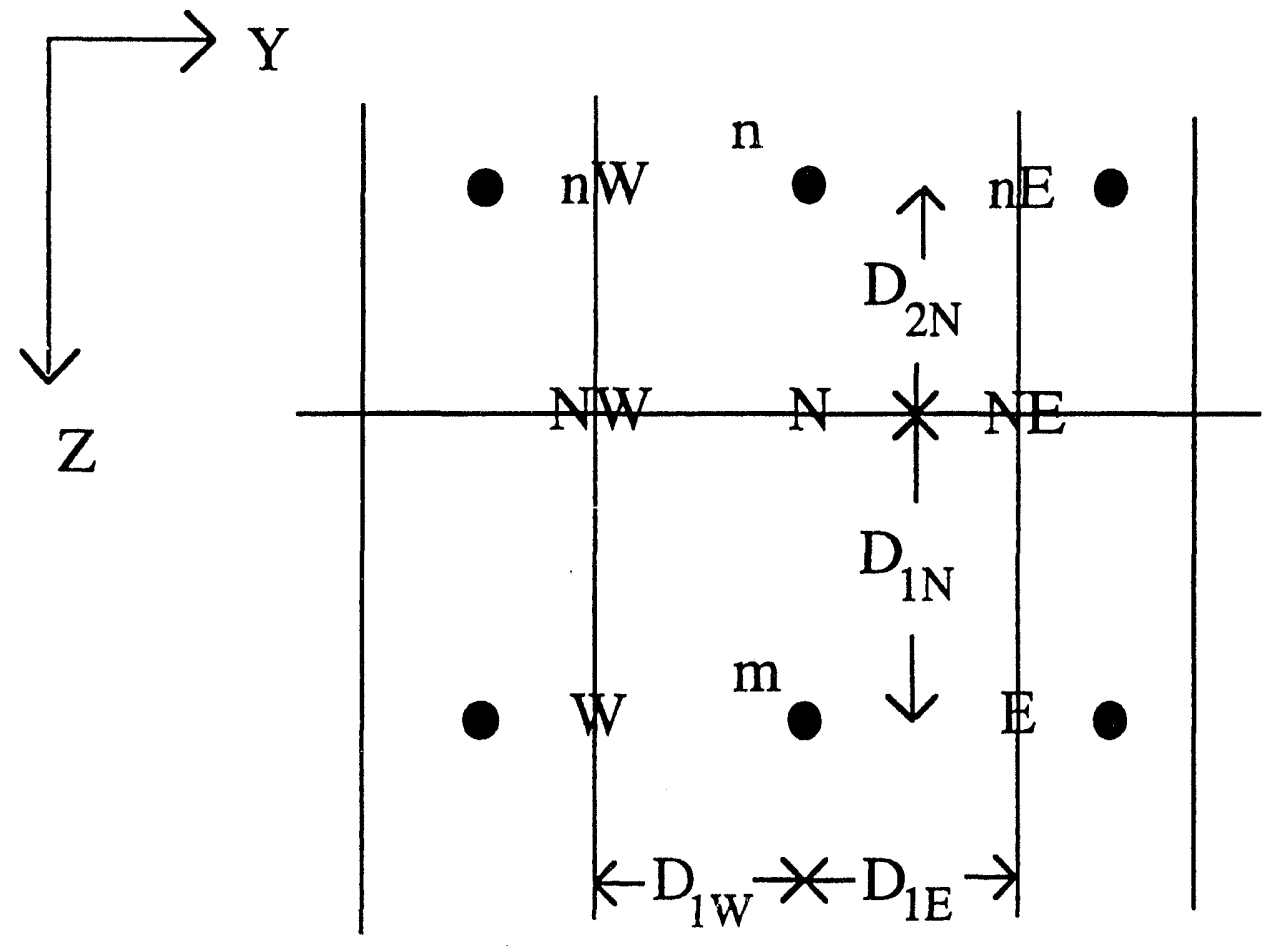

Fig. 3. Interfacial distances relevant to the interpolation of velocity and gradient vectors onto interface $\mathrm{N}$.

$$
\mathrm{U}_{\mathrm{NE}}=\frac{\mathrm{U}_{\mathrm{nE}} \mathrm{D}_{1 \mathrm{~N}}+\mathrm{U}_{\mathrm{E}} \mathrm{D}_{2 \mathrm{~N}}}{\mathrm{D}_{1 \mathrm{~N}}+\mathrm{D}_{2 \mathrm{~N}}}
$$

are the vertex values of the velocity component. Note that DISF requires that nodes be centered in their grid blocks $\left(D_{1 E}=D_{1 W}\right)$. It is apparent from Eqs. 10-12 that the $Y$ component of velocity at interface $\mathrm{N}$ is dependent on thermodynamic conditions (primary variables) at all of the six grid blocks marked in Fig. 3. The Z-component of velocity is known explicitly at $\mathrm{N}$ from data given for grid blocks $\mathrm{n}$ and $\mathrm{m}$ and no interpolation is required. Analogous interpolation is made at all other interfaces. The component of the solute concentration gradient vector perpendicular to the interface is calculated as a first order finite difference by taking the difference of the nodal values divided by the connection distance. After these direct gradient components are calculated, they are interpolated onto 
the interfaces in the same manner as the velocity vectors (Eq. 10). It is important to note that the interpolation scheme used in DISF requires that all computational domains be at least 2 grid blocks on each side in the $\mathrm{Y}$ - and Z-directions. The smallest domain compatible with DISF consists of four grid blocks, two in the Y-direction and two in the Z-direction.

As stated above, at each interface one component of the velocity vector, and one component of the gradient vector are known directly, thus obviating the need for in terpolation (Fig. 4).

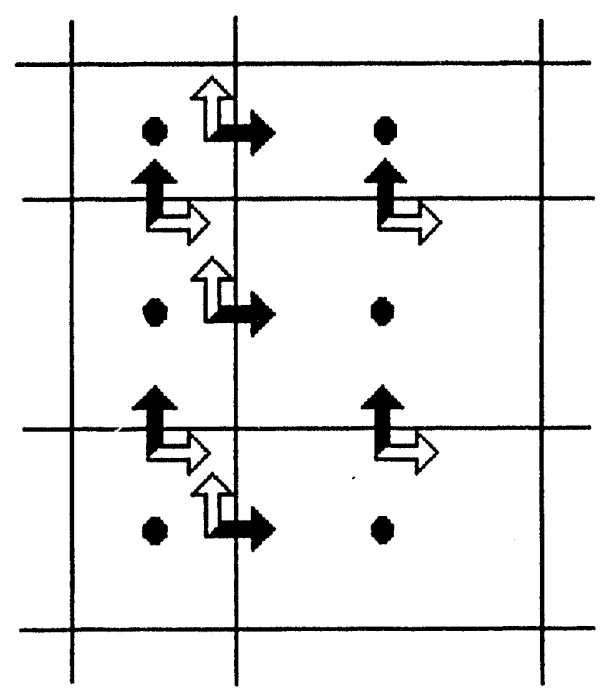

Fig. 4. Velocity or concentration gradient vector components at grid block interfaces. Filled (black) components of velocity are known directly. Unfilled (white) components must be interpolated. Filled (black) components of concentration gradient vectors can be calculated directly by differencing of the known composition values at the nodes (solid circles) and dividing by their connection distances. Unfilled (white) components must be interpolated from the interfaces where they can be calculated directly. 
In the standard TOUGH2 methodology, the flow rate across the interface between two grid blocks depends only on the primary variables and properties of the two connected grid blocks. The flow term thus gives rise to two NEQ $\times$ NEQ submatrices of derivatives in the Jacobian matrix located in the off-diagonal locations corresponding to the two grid blocks involved. Because of the interpolation in T2DM, six grid blocks are involved in calculating the dispersive fluxes. This is shown schematically in Fig. 5 where we consider the dispersive flux across the interface $\mathrm{N}$ (shown by dark line). The direct vector components at interface $\mathrm{N}$ (normal to the interface) depend only on primary variables in the grid blocks shown by the cross-hatch pattern; the interpolated indirect components (parallel to the interface) depend on primary variables in all six grid blocks shown. Because the dispersive flux depends on the full vectors, which are composed of both direct and interpolated components, T2DM generates for each interface six NEQ x NEQ submatrices in the Jacobian matrix. The increased number of non-zero terms in the Jacobian matrix increases storage requirements and execution times of the linear equation solver relative to the standard TOUGH2. To accommodate the increased storage requirements, the user should set the parameter MNCON equal to three times the actual maximum number of connections. DISF places values into the Jacobian in some of the same places as MULTI, as well as placing multiple values into the same locations in the Jacobian matrix. The linear equation solver MA28 will efficiently sum these duplicate elements and give an informative warning message that appears in the file LINEQ produced during execution.

In some applications, the extra terms in the Jacobian arising from the dispersive flux dependence on neighbor grid blocks are less important than the direct terms arising from the two connected grid blocks. These extra terms can be optionally neglected by appropriate specification of parameter $\mathrm{MOP}(23)$ (see section 4.2). Neglecting these terms produces a sparser though less-accurate Jacobian matrix which may limit convergence rates and time-step sizes. However, the linear equations are solved faster for the sparser Jacobian, which for some flow problems may compensate for the decreased time-step size. 


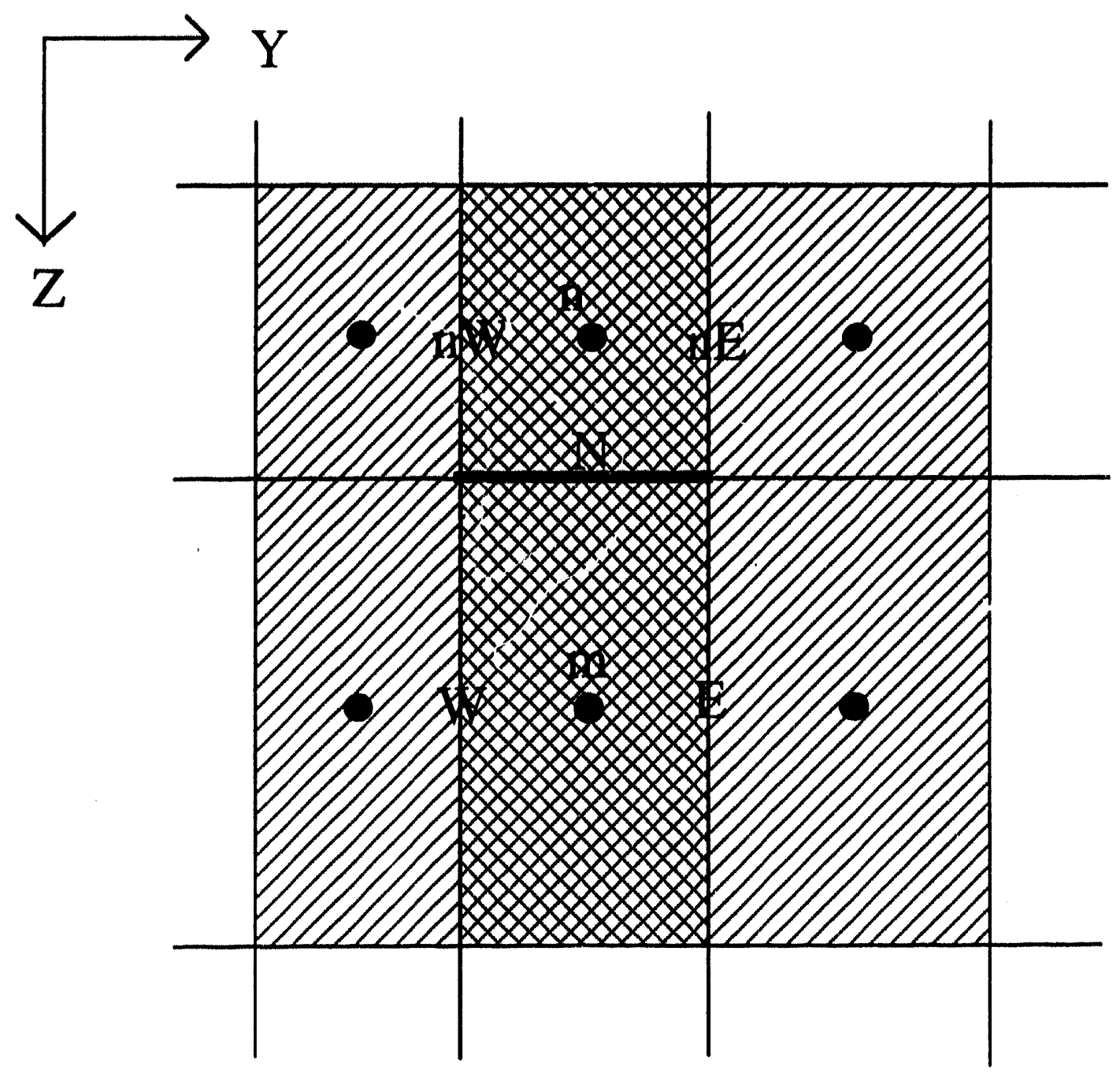

Fig. 5. The grid blocks involved in calculating dispersive flux at interface $N$. The direct terms arise from the connected grid blocks ( $m$ and $n$ ) shown by the cross-hatch pattern.

The indirect terms arise from the neighbor grid blocks shown by the diagonal line pattern. 


\subsection{Boundary Conditions}

In standard TOUGH2 methodology, the calculation domain boundary coincides with the flow domain boundary. For many transport prcblems, it is useful to define a flow domain consisting of grid blocks that are a subset of the grid blocks comprising the calculation domain. This allows a flexible definition of concentration and Darcy velocity conditions on the flow domain boundary. For example, if one wishes to assign concentration boundary conditions, this can be accomplished by assigning appropriate brine mass factions to grid blocks outside of the flow domain, and giving these grid blocks very large volumes, say $1 . e 40 \mathrm{~m}^{3}$, so inat their conditions will remain unchanged. An example of such boundary conditions is given in the variable density transport problem below. A sketch of a flow domain within the calculation domain is shown in Fig. 6.

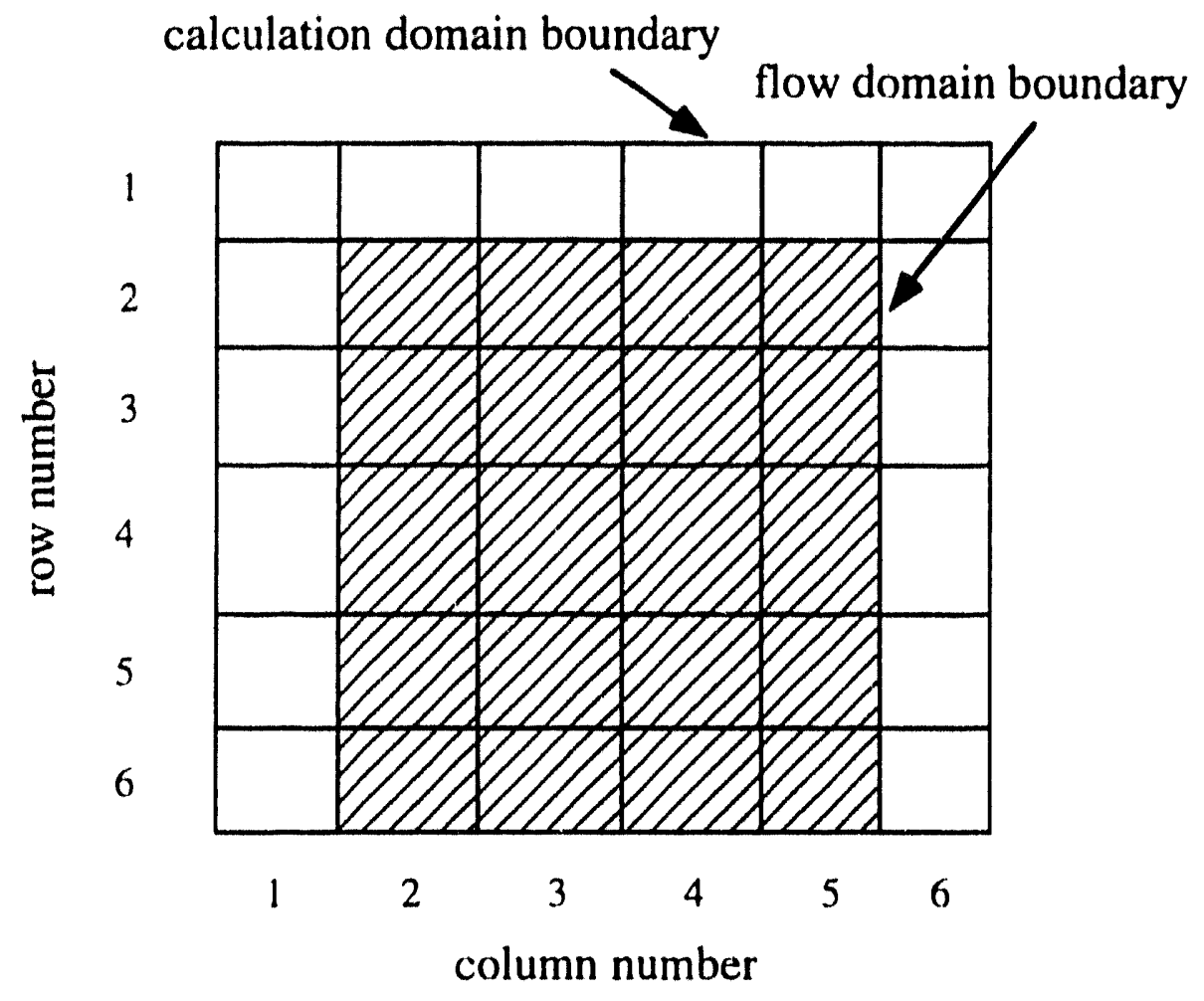

Fig. 6. Sketch of flow domain (filled with diagonal lines) within calculation domain. On the bottom, the flow and calculation domains coincide. 
The dimensions of the flow domain are specified in the input file by the parameters NFBL, NFBR, NFBT, and NFBB where NFBL stands for the column (or row) number of the flow boundary on the left and similarly for the other parameter names where the $R, T$ and $B$ stand for right, top, and bottom, respectively (see also section 4.2). For exampic, in Fig. $6, \mathrm{NFBL}=2, \mathrm{NFBR}=5, \mathrm{NFBT}=2$, and $\mathrm{NFBB}=6$. If these parameters are not specified, they will be defaulted such that the flow domain is the same as the calculation domain.

Calculation of dispersive fluxes requires full vector components of the Darcy velocity and brine mass fraction vectors, $u$ and $\nabla X$ (see Eq. 9). As discussed above, vector components perpendicular to an interface between grid blocks (the "direct" components) are always calculated directly from thermodynamic parameters of the neighboring grid blocks, using first-order finite difference expressions. This procedure is followed regardless of whether the interface is in the interior or on the boundary of the flow domain. (Note that when the flow domain coincides with the calculation domain, no-flow interfaces will be present at the boundary, so that the question of how to calculate direct vector components does not arise.) Vector components parallel to an interface must be obtained by interpolation (see Figs. 3-5). Special considerations are required for interpolating these "indirect" components near the flow domain boundary, where some of the vector components needed for interpolation are not available. It is necessary, therefore, to assign the missing components in a way that will properly describe the applicable boundary conditions.

There are two cases for the missing vector component that must be considered: (i) a vector component situated right on the boundary and oriented parallel to it (Fig. 7); and (ii) a vector component perpendicular to the boundary at an interface in immediate proximity to the boundary (Fig. 8). In either case, only two of the four vector components needed for interpolation can be determined from parameters within the flow domain. Several options are available for assigning the two "missing" components. The options allow 


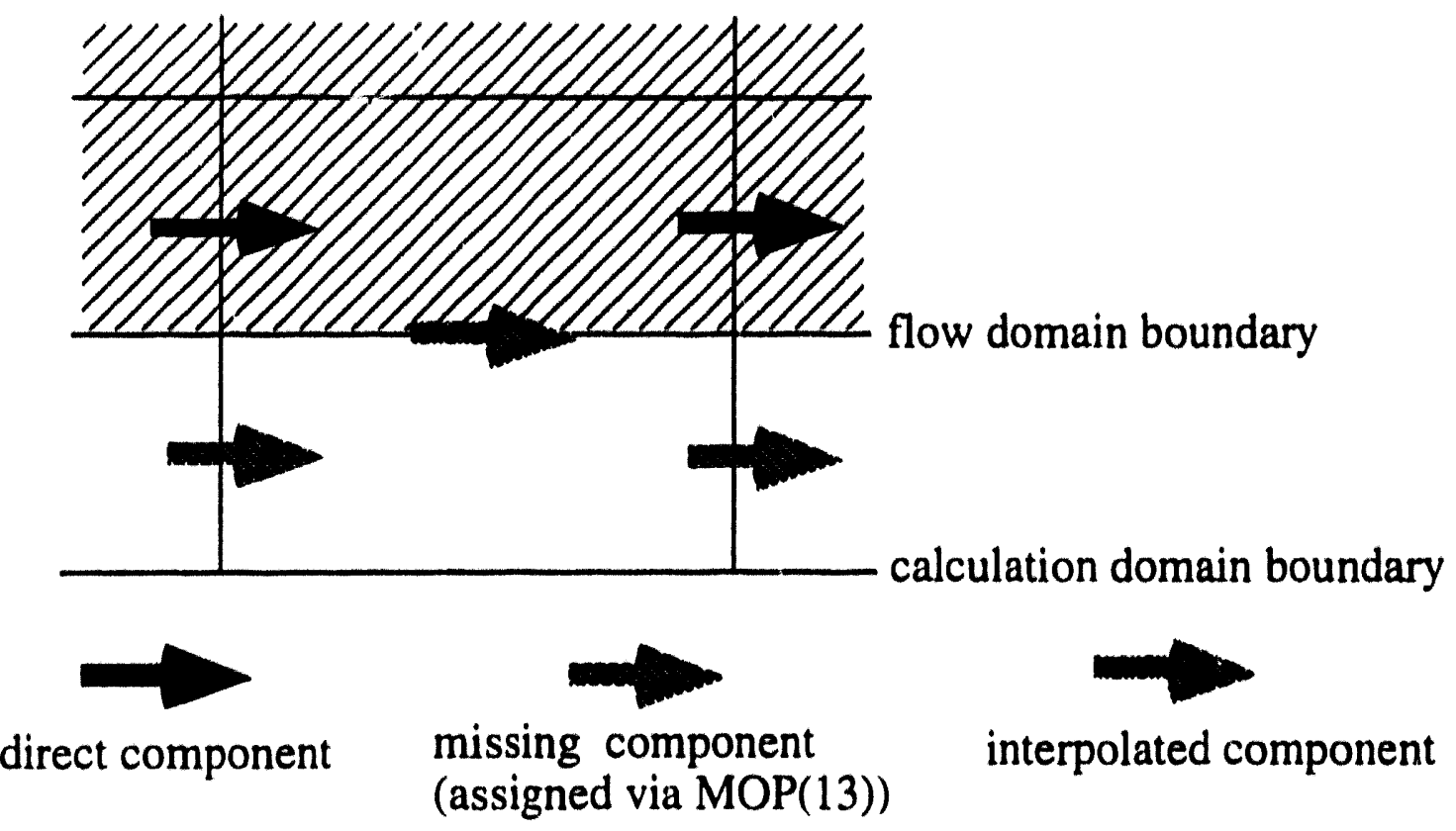

Fig. 7. Interpolation of a parallel vector component at the flow domain boundary.

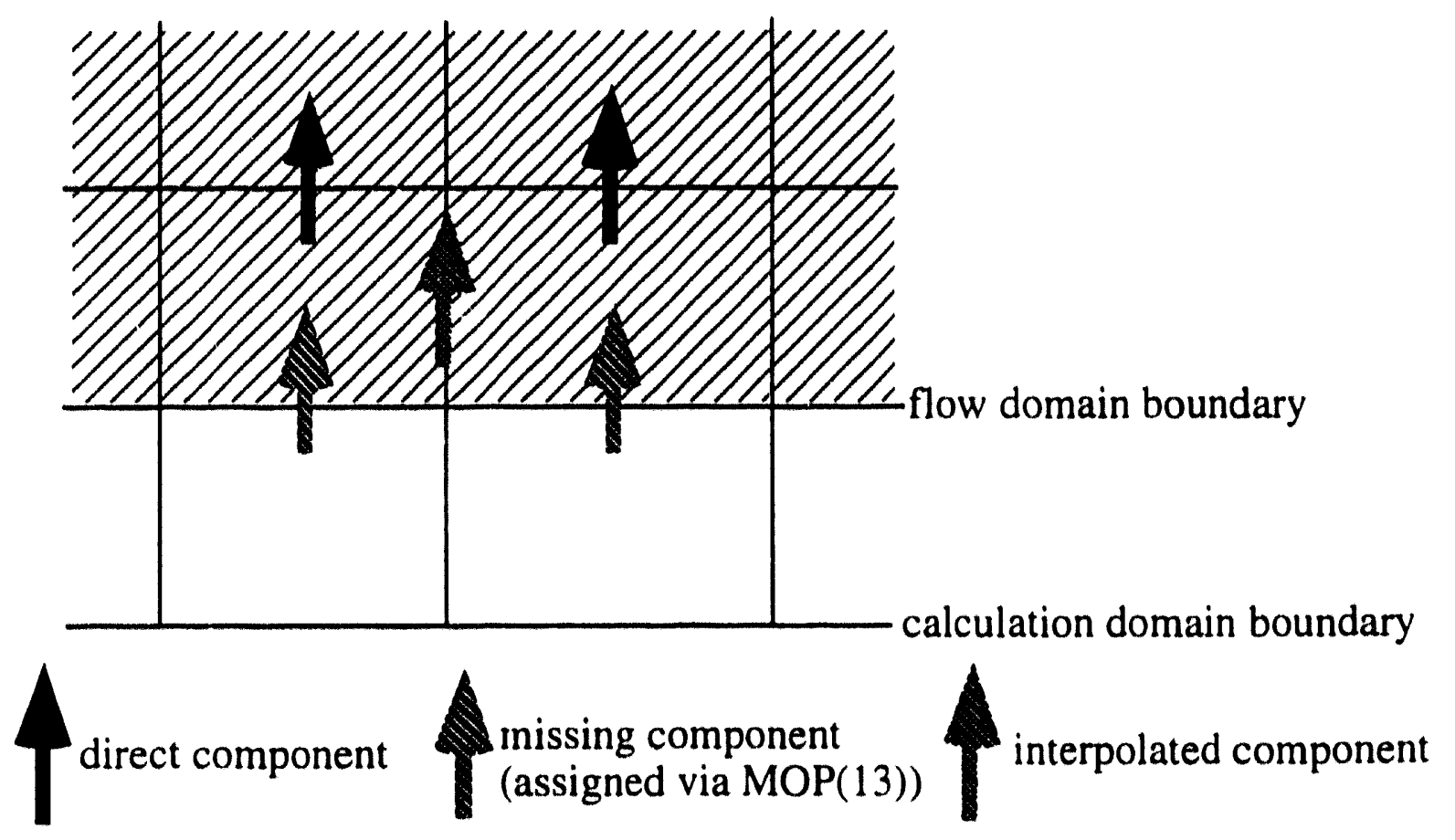

Fig. 8. Interpolation of a perpendicular vector component at the flow domain boundary. 
flexibility in modeling different physical situations and are selected by means of the parameter $\mathrm{MOP}(13)$, as follows. For $\mathrm{MOP}(13)=0$, the missing vector components for both Darcy velocity and brine mass fraction are assigned to be zero. For $\operatorname{MOP}(13)=3$, the missing vector components are assigned to be equal to their "nearest neighbors" within the flow domain, i.e., equal to the vector components shown in solid black in Figs. 7 and 8. This option effectively causes the indirect components to be interpolated from just the two nearest neighbors within the flow domain. Mixed options can be selected with $\operatorname{MOP}(13)=$ 1 or 2 . For $\operatorname{MOP}(13)=1$, the missing Darcy velocity components are assigned to be zero, and the missing brine mass fraction gradient components are assigned equal to their nearest neighbor values. Conversely, for $\operatorname{MOP}(13)=2$ the missing Darcy velocity components are assigned to their nearest neighbor values, while the missing brine mass fraction gradient components are set to zero.

We emphasize that the only effect of MOP(13) is to select interpolation options for the indirect vector components near the flow domain boundary, as shown in Figures 7 and 8 . Furthermore, the assignment of missing vector components for interpolation is the same, regardless of whether or not the calculation domain coincides with the flow domain.

\subsection{Restrictions on the Mesh}

In order to make the interpolation algorithm simple and robust, the MESH must be a rectangular $\mathrm{Y}-\mathrm{Z}$ grid listed by-columns as will be generated internally by TOUGH2 by appropriate specification using MESHMAKER. The nodes must be centered in the grid blocks. The feature of TOUGH2 which allows users to make grid blocks inactive by giving them zero volume and listing them at the end of the ELEME block is not compatible with T2DM. Instead, inactive grid blocks should be defined by giving them effectively infinite volumes $\left(e . g, 1 . e 40 \mathrm{~m}^{3}\right.$ ) by editing the MESH file. Future releases of TOUGH2 will provide more flexible options for creating inactive grid blocks. The restriction to rectangular domains should not prove limiting since any arbitrary properties and volumes 
can be given to the grid blocks and their connections to create an arbitrarily shaped region of interest within a rectangular set of grid blocks.

\subsection{Interface Conductivity}

The molecular diffusion terms in Eqs. 5 and 6 require interface values of the product $\phi S \tau d$. Because the molecular diffusivity $d$ is spatially invariant, the effective conductivity term that must be approximated at each interface is actually the product $\phi S \tau$. We have used harmonic weighting to approximate the effective interface conductivity terms.

The harmonic weighting scheme can be derived by conservation of flux across the interface, in complete analogy to permeability weighting in Darcy flow in heterogeneous porous media. Specifically, consider two grid blocks with connection distances $D_{1 N}$ and $\mathrm{D}_{2 \mathrm{~N}}$ (Fig. 3). If the concentration at the nodes is given by $C_{m}$ and $C_{n}$, we may write

$$
C_{n}-C_{m}=C_{n}-C_{N}+C_{N}-C_{m}
$$

We can furthermore write the diffusive flux at interface $\mathrm{N}$ as

$$
j_{N}=\left[-\rho \phi S_{\beta} \tau d \nabla C\right]_{N}=\rho_{N} K_{N} d \frac{\left(C_{n}-C_{m}\right)}{\left(D_{1 N}+D_{2 N}\right)}
$$

where $K_{N}$ is the unknown interface value of the product $\phi S \tau$. For simplicity, we assume for the time being that the density is constant and consider only the interface conductivity term $K_{N}$. Because the flux is continuous across the interface, the fluxes from $\mathrm{m}$ to $\mathrm{N}$ and from $\mathrm{N}$ to $\mathrm{n}$ are equal to the flux across the interface $\mathrm{N}, j_{N}$ :

$$
\begin{aligned}
& \rho_{N} d\left(C_{n}-C_{m}\right)=\frac{j_{N}}{K_{N}}\left(D_{1 N}+D_{2 N}\right) \\
& \rho_{N} d\left(C_{n}-C_{N}\right)=\frac{j_{N}}{K_{n}} D_{2 N}
\end{aligned}
$$




$$
\rho_{N d}\left(C_{N} \cdot C_{m}\right)=\frac{j_{N}}{K_{m}} D_{1 N}
$$

Subitituting Eqs. 15-17 into 13 and canceling common terms gives rise to the harmonic weighting scheme for the interface conductivity $K_{N}$ :

$$
\frac{D_{1 N}+D_{2 N}}{K_{N}}=\frac{D_{1 N}}{K_{m}}+\frac{D_{2 N}}{K_{n}}
$$

or

$$
K_{N}=\frac{K_{m} K_{n}\left(D_{1 N}+D_{2 N}\right)}{K_{n} D_{1 N}+K_{m} D_{2 N}}
$$

For molecular diffusion, the interface fluid density, assumed constant for simplicity in the above derivation, is taken to be the average fluid density in the two grid blocks. For hydrodynamic dispersion, upstream weighting of the interface density is used.

\section{Using the Dispersion Module}

\subsection{Compilation}

The dispersion module (T2DM) cunsists of a main program (TOUGH2) and eight subroutines: CYCIT, MULTI, DISF, DOT, LINEQ, OUTDF, MESHM, and GXYZ. Table 1, taken from a TOUGH2 output file, shows revised TOUGH2 routines marked with a "D", while the new subroutines for dispersion are ilı licated by an exclamation point. DISF is the new subroutine which calculates the dispersive flux and augments the flux term as shown in Eq. 9. There is a small companion subroutine to DISF called DOT, which calculates the components of the unit normal vector to the boundaries of the grid blocks. All of the above source codes are concatenated together as the file T2DM. 
Table 1. Summary of program units in T2DM.

\begin{tabular}{|c|c|c|c|c|}
\hline WNIT & venston & Dart: & & conowrs \\
\hline 10 & 1.0 & is Amst & 1911 & 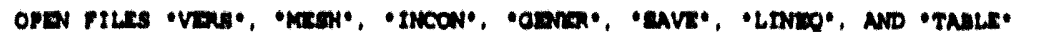 \\
\hline TоUона & 1.00 & $12 \operatorname{ran}$ & 1992 & men moonur \\
\hline INRT & 1.0 & 1) Nart & 1891 & 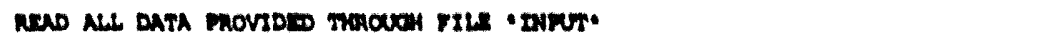 \\
\hline manos & 1.00 & $17 \operatorname{rar}$ & 1992 & 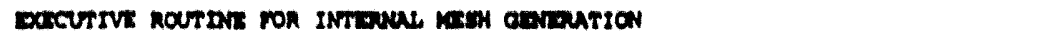 \\
\hline 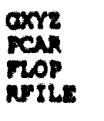 & $\begin{array}{l}1.00 \\
1.0 \\
1.0 \\
1.0\end{array}$ & $\begin{array}{l}\text { jo rwe } \\
29 \text { wach } \\
\text { if APMIL } \\
\text { as AMkIL }\end{array}$ & $\begin{array}{l}1992 \\
1991 \\
1991 \\
1991\end{array}$ & 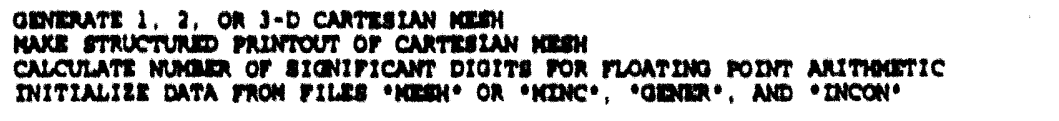 \\
\hline crert & 1.10 & OS maY & 1913 & 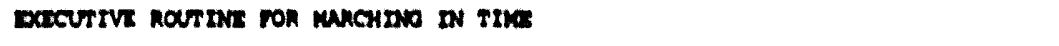 \\
\hline 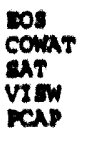 & $\begin{array}{l}0.11 \\
1.0 \\
1.0 \\
1.0 \\
1.0\end{array}$ & 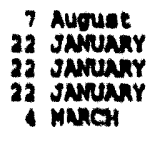 & $\begin{array}{l}1992 \\
1990 \\
1990 \\
1990 \\
1991\end{array}$ & 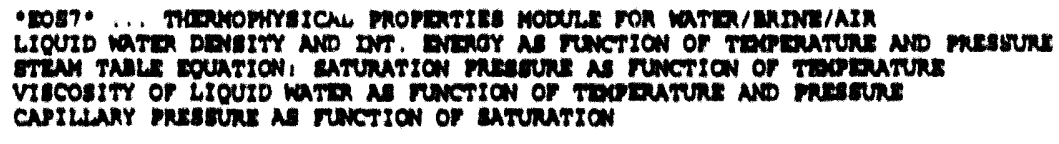 \\
\hline when & 1.0 & $16 \pi t y$ & 1921 & 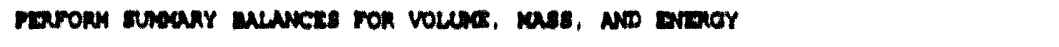 \\
\hline murt & 1.10 & 2A INALIL & 1993 & 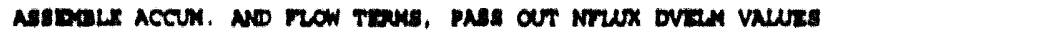 \\
\hline$\infty$ & 0.61 & 22. JANunkY & 1993 & 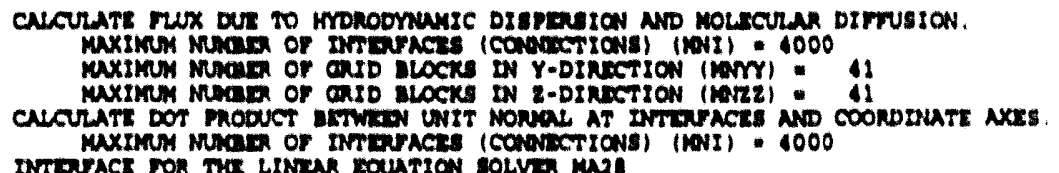 \\
\hline 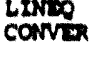 & 1.10 & of ming & 1993 & 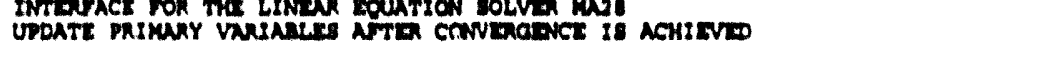 \\
\hline our & 1.0 & I AUCust & 1991 & 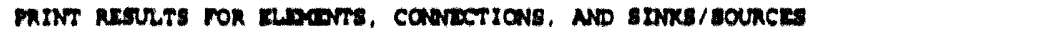 \\
\hline $\begin{array}{l}\text { arror } \\
\text { Whiri }\end{array}$ & 0.31 & $\begin{array}{l}17 \text { rave } \\
22 \text { JANunkY }\end{array}$ & 1992 & 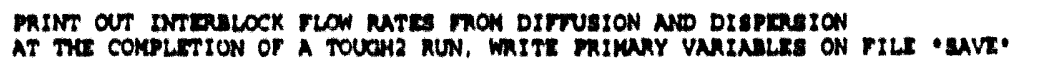 \\
\hline
\end{tabular}

The subroutines comprising T2DM must be compiled and linked in front of the standard TOUGH2 subroutines, using the EOS7 equation-of-state module for saline brines with gas (Pruess, 1991). A typical compilation and linking instruction on an IBM RS/6000, for example, will be as follows:

xlf -O -qautodbl=dblpad $22 d m . f(2 m . f$ t2f.f eos 7 .f meshm.f ma28.f

where the italics indicate the new dispersion module consisting of all of its source files.

During linking, warnings indicating duplicate subroutine names may appear on some 
computers, but the linker will use the first subroutine with a given name ${ }^{\dagger}$. That is the reason for linking the T2DM subroutines in front of the standard subroutines. In Appendix I we present a small test problem useful for checking proper installation of T2DM.

\subsection{Input Formats}

Input to DISF is given through the PARAM and SELEC blocks of TOUGH2 as follows:

PARAM.1 format(212, 314, 24I1, 2E10.4)

NOITE, KDATA, MCYC, MSEC, MCYPR, (MOP(I), I = I, 24), DIFFO, TEXP

see TOUGH report (Pruess, 1987) and informative TOUGH2 printout for description of all of the above parameters except the following:

MOP(8) specifies additional diagnostic printout from T2DM. Amount of printout increases with larger value of $\operatorname{MOP}(8)$.

MOP(13) determines assignments of boundary vector components for interpolation. Affects both the flow domain boundary and calculation domain boundary identically.

0: velocity and concentration gradient vectors are zero.

1: vel xcity is zero; concentration gradient is nearest neighbor.

2: velocity is nearest neighbor; concentration gradient is zero.

3: velocity and concentration gradient are nearest neighbor.

\footnotetext{
† Some linkers produce fatal errors when encountering duplicate names. In such a case, the TOUGH 2 routines of which modified versions are included in $2 \mathrm{dm}$. $\mathrm{f}$ must be removed from the Version 1.0 TOUGH2 files.
} 
MOP(23)

handles treatment of non-direct terms in the Jacobian.

0 : include non-direct terms.

1: neglect non-direct terms.

SELEC keyword to introduce a data block with reference brine, geometry and dispersion data.

SELEC.1 format(8I5)

IE(1), NGBINP(1),NGBINP(2),NGBINP(3),NFBL,NFBR,NFBT,NFBB

IE(1) set equal to 4 to read four additional data records of brine, geometry, and dispersion data.

NGBINP(1) number of grid blocks in $X$ (must always be equal to 1 ).

NGBINP(2) number of grid blocks in Y.

NGBINP(3) number of grid blocks in Z.

NFBL. number of the first ("left") column of grid blocks within the flow domain (defaults to 1 if zero or blank).

NFBR number of the last ("right") column of grid blocks within the flow domain (defaults to NGBINP(2) if zero or blank).

NFBT number of the first ("top") row of grid blocks within the flow domain (defaults to 1 if zero or blank).

NFBB number of the last ("bottom") row of grid blocks within the flow domain (defaults to NGBINP(3) if zero or blank). 
SELEC.2 format(3E10.4)

$$
\begin{array}{ll}
P_{0}, T_{0}, \rho_{b} & \\
P_{0} & \text { reference pressure, } P a . \\
T_{0} & \text { reference temperature, }{ }^{\circ} \mathrm{C} . \\
\rho_{b} & \text { brine density at }\left(P_{0}, T_{0}\right), \mathrm{kg} \mathrm{m}^{-3} .
\end{array}
$$

For $P_{0}, T_{0}$, and $\rho_{b}$ equal to zero or blank, default values of $P_{0}=1 \times$ $10^{5} \mathrm{~Pa}, \mathrm{~T}_{0}=25^{\circ} \mathrm{C}, \rho_{\mathrm{b}}=1185.1 \mathrm{~kg} \mathrm{~m}^{-3}$ will be used. If $\mathrm{P}_{0}<0$, brine will have the same thermophysical properties as pure water, allowing the brine component to behave as a tracer component (see EOS7 User's Guide; Pruess, 1991)

SELEC.3 format(3E10.4)

$v(i), i=1,3$

$v(\mathrm{i})$

coefficients for salinity correction in aqueous phase viscosity (see EOS7 User's Guide; Pruess, 1991). For v(i) $=0$, default values will be used. Specification of pure water in record SELEC.2 will override viscosity specifications.

SELEC.4 format(2E10.4)

ALPHAT, ALPHAL ALPHAT transverse dispersivity, $\mathrm{m}$.

ALPHAL longitudinal dispersivity, $m$.

SELEC.5 format(6E10.4)

FDDIAG(NP,NK), NK=1,3; NP=1,2

FDDIAG(NP,NK) Molecular diffusivities: first parameters for three component diffusivities in phase 1, then parameters for three component 
diffusivities in phase 2 (see Table 2). Note that the tortuosity of each material in the ROCK block of the input file must be set to some non-zero value to model molecular diffusion effects.

Table 2. Molecular diffusivities for two phases and three components.

\begin{tabular}{lllll}
\hline phase & component & $(\mathrm{NP}, \mathrm{NK})$ & input & units \\
\hline gaseous & water & $(1,1)$ & FDDIAG(1,1) & $\mathrm{m}^{2} \mathrm{~s}^{-1}$ \\
& brine & $(1,2)$ & FDDIAG(1,2) & $\mathrm{m}^{2} \mathrm{~s}^{-1}$ \\
& air & $(1,3)$ & FDDIAG(1,3) & $\mathrm{m}^{2} \mathrm{~s}^{-1}$ \\
aqueous & water & $(2,1)$ & FDDIAG(2,1) & $\mathrm{m}^{2} \mathrm{~s}^{-1}$ \\
& brine & $(2,2)$ & $\operatorname{FDDIAG}(2,2)$ & $\mathrm{m}^{2} \mathrm{~s}^{-1}$ \\
& air & $(2,3)$ & FDDIAG(2,3) & $\mathrm{m}^{2} \mathrm{~s}^{-1}$ \\
\hline
\end{tabular}

\section{Sample Problems}

\subsection{One-Dimensional Transport with Dispersion}

This problem considers the one-dimensional transport of a tracer in a homogeneous saturated porous medium with a steady flow field of $0.1 \mathrm{~m} /$ day pore velocity. A tracer solute is introduced at the left-hand side, as shown in Fig. 9. Transport is by advection and hydrodynamic dispersion. An analytical solution for this problem is given in Javandel et al. (1984). The TOUGH2 solution of this problem is accomplished by specifying constant pressure boundary conditions which will give rise to a steady-state flow field with $0.1 \mathrm{~m} /$ day pore velocity $(1.157 \mathrm{e}-6 \mathrm{~m} / \mathrm{s})$. The TOUGH2 input file is shown in Fig. 10. Note that the brine is made a tracer chemical component, with identical thermophysical 


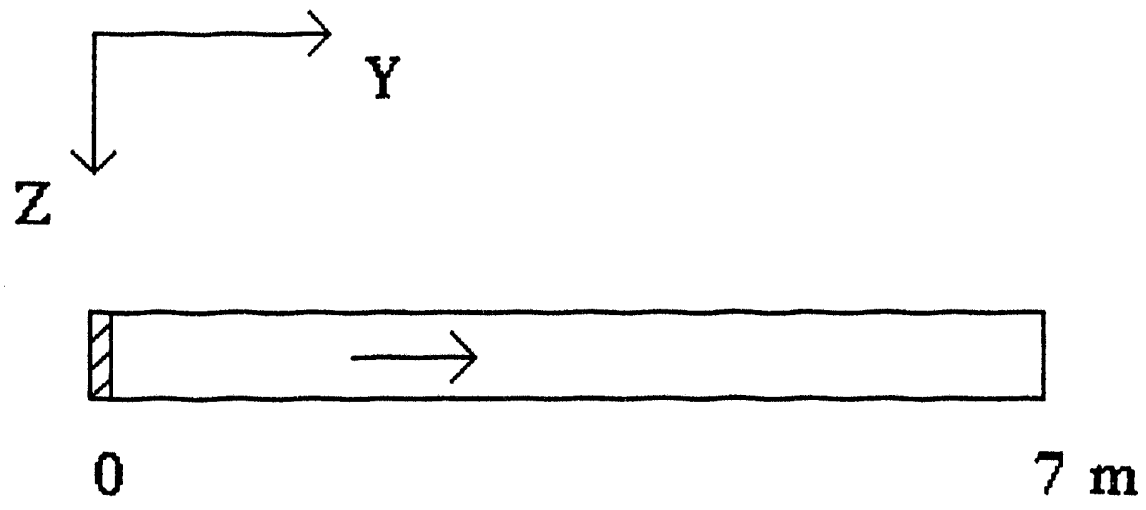

Fig. 9. Domain for one-dimensional dispersion problem.

properties as pure water, by specification of the reference pressure as a negative number. Also note that in keeping with the requirements of the interpolation scheme in DISF, the domain is pseudo-one-dimensional, and actually is two grid blocks wide. Molecular diffusivity is set to zero for all components.

The MESH file was created with MESHMAKER and then edited "by hand" to give the left- and right-hand side columns of grid blocks effectively infinite volumes so that their pressures and mass fractions of solute are effectively constant. Then appropriate initial conditions are chosen such that brine is injected in the left-hand side (grid blocks A11 1 and A21 1) and the pressure difference between the left and right sides gives rise to the correct velocity field. These initial conditions are shown in the input file in Fig. 10.

Results for the transport of the brine tracer at $t=20$ days are shown in Figs. 11 and 12. The analytical solution is shown by the solid lines along with results for three different discretizations (the coarse discretization of Fig. 10 and two higher-resolution grids). The data points on Figs. 11 and 12 are at the nodal points and show the values of brine concentration. Results for the three different discretizations show the expected trend toward diminished numerical dispersion and improved agreement with the analytical 


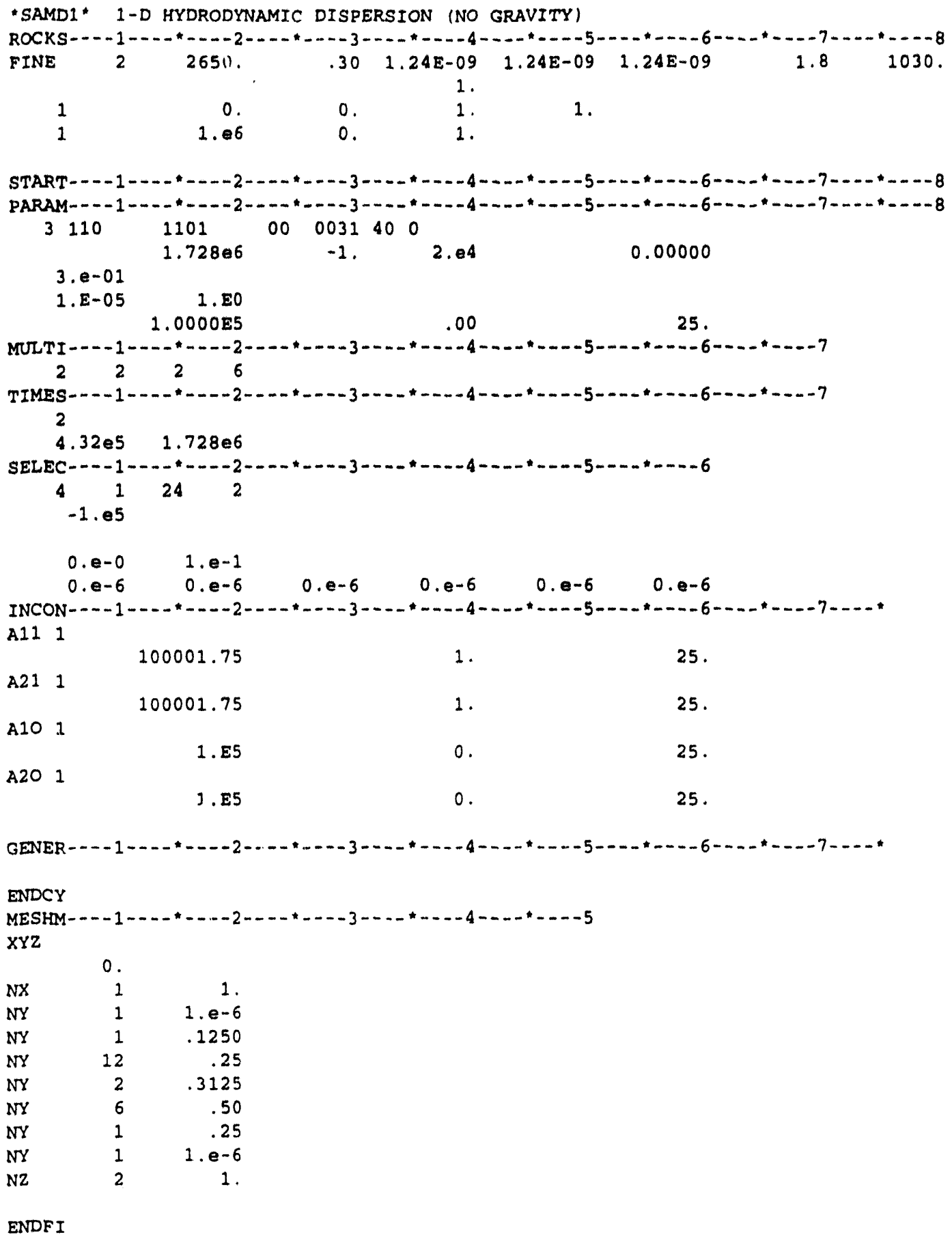

Fig. 10. Input file for the one-dimensional dispersion problem. 


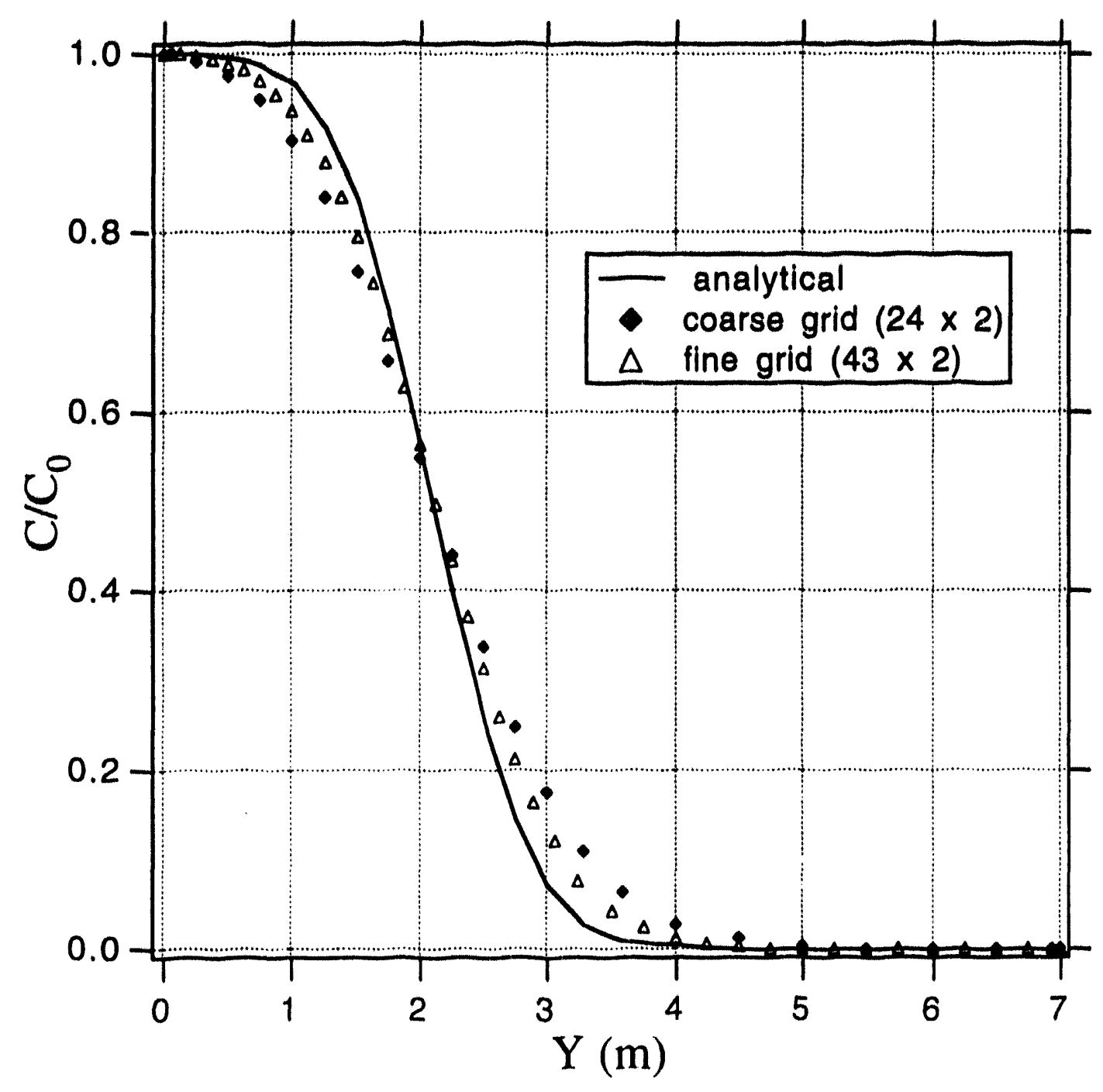

Fig. 11. Brine concentration for one-dimensional dispersion problem at $t=20$ days. The solid line represents the analytical result, open triangles show numerical results with the coarse $(24 \times 2)$ grid, and filled diamonds show numerical results for the fine $(43 \times 2)$ grid. 


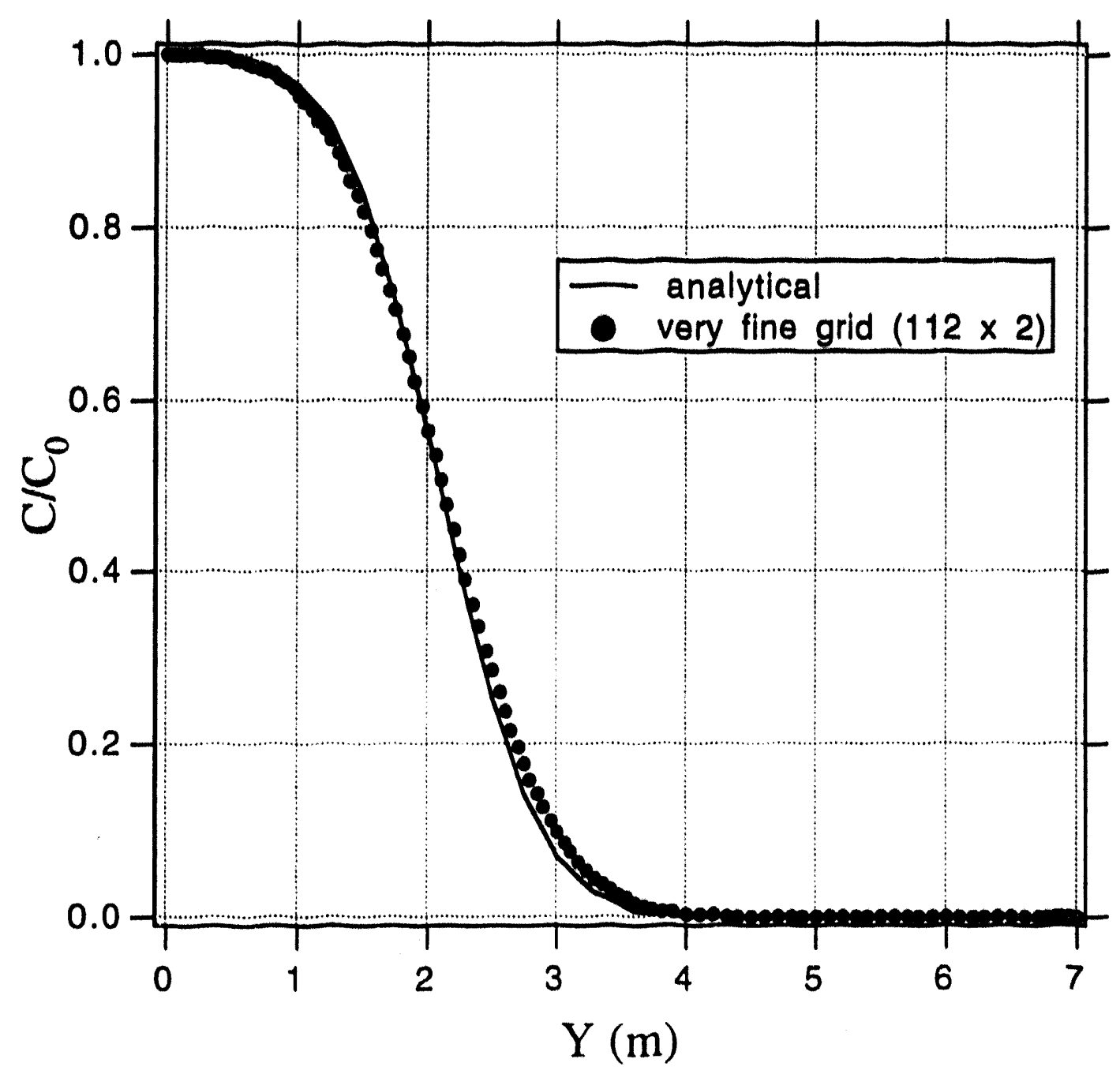

Fig. 12. One-dimensional tracer transport results at $t=20$ days. 
solution as the grid becomes finer. To cherk the convergence of the dispersion model as the effects of numerical dispersion are decreased, a very fine grid (112 grid blocks in Ydirection) was used for this one-dimensional problem. The calculated front at $t=20$ days for this very fine grid is plotted in Fig. 12 and shows very good agreement with the analytical result. By using the automatic time-step control option of TOUGH2 (MOP(16)) and varying the maximum time step size through input parameter DELTMX, the effects of time discretization errors were investigated and determined to be of secondary importance relative to the space discretization errors for causing the observed numerical dispersion. Results plotted in Fig. 12 were calculated with DELTMX = 2.e4 seconds. 


\subsection{Two-Dimensional Transport with Dispersion}

This problem considers the two-dimensional transport and dispersion of a tracer introduced into the left-hand side of a homogeneous isotropic, saturated porous medium with a steady-state flow field from left to right of $0.1 \mathrm{~m} /$ day pore velocity. The tracer is introduced along a line source of length $a=0.5 \mathrm{~m}$ as shown in Fig. 13. An analytical

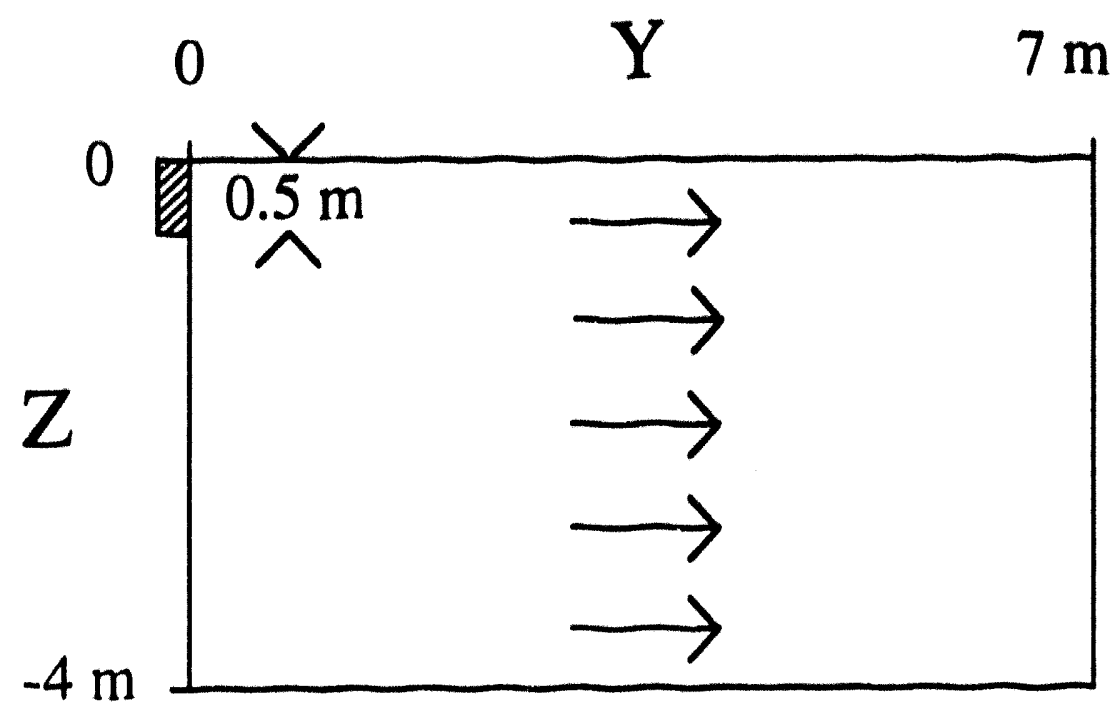

Fig. 13. Two-dimensional tracer transport problem. Source in upper left-hand corner is of length $0.5 \mathrm{~m}$. Flow field is uniform and steady. There is no gravity in the problem.

solution for this problem is given in Javandel et al. (1984) along with a code for calculating the tracer concentration. The two discretizations used are shown in Fig. 14. Note that the Y-direction discretization shown in Fig. 14a is the same as for the one-dimensional transport problem of Fig. 9. The input file for the two-dimensional problem is given in Fig. 15. Note that a negative reference pressure is specified in the input file (SELEC.2) making the thermophysical properties of the brine component equal to the properties of pure water. In this way, the brine is effectively a tracer component. Transverse and longitudinal dispersivities are .025 and $.1 \mathrm{~m}$, respectively. A maximum time step size of 

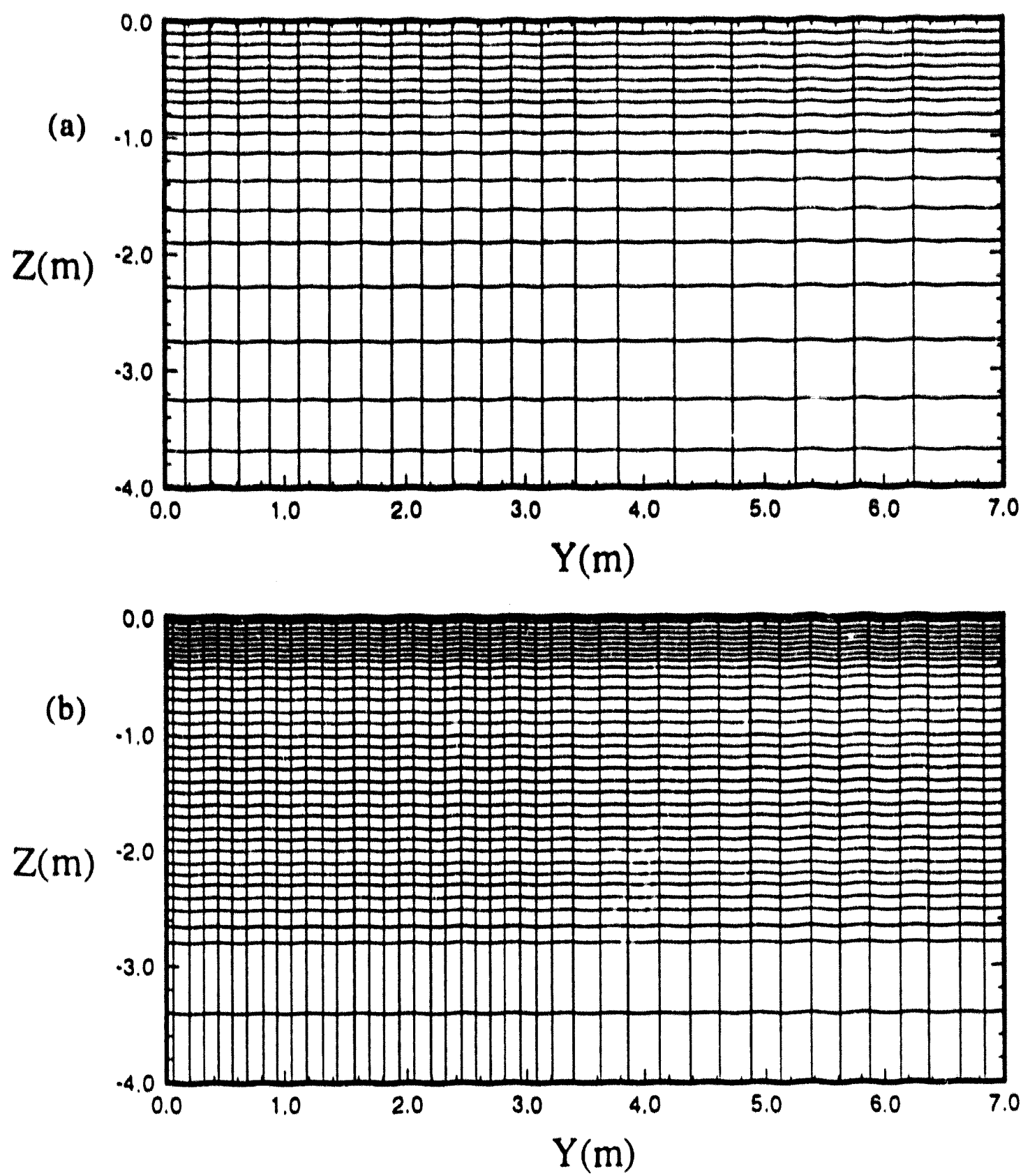

Fig. 14. (a) Coarse and (b) fine discretizations for the two-dimensional tracer transport. Note that there are two columns of grid blocks on the left-and right-hand sides which are of effectively infinite volume which are not shown. 


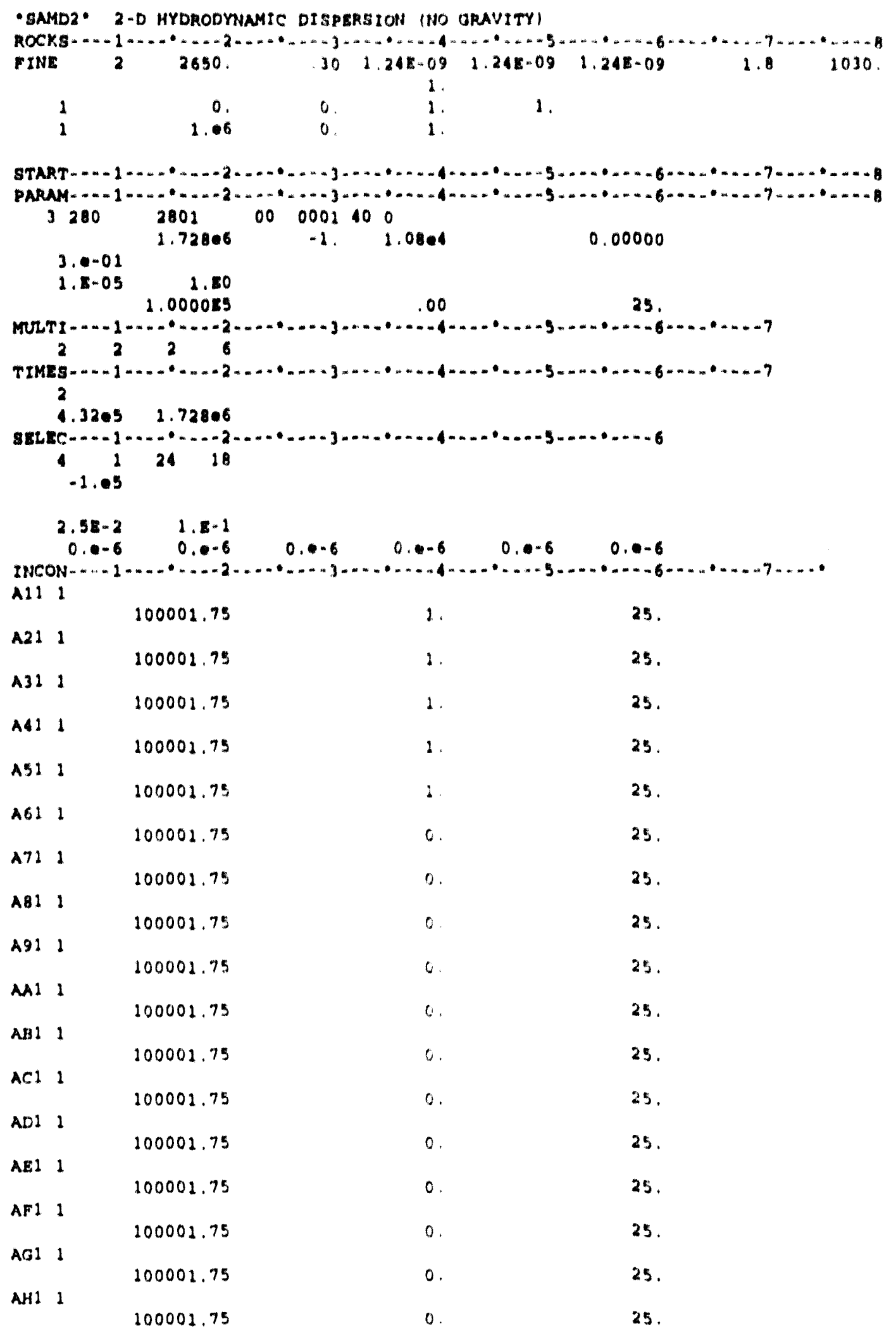

Fig. 15. Input file for the two-dimensional transport problem. 


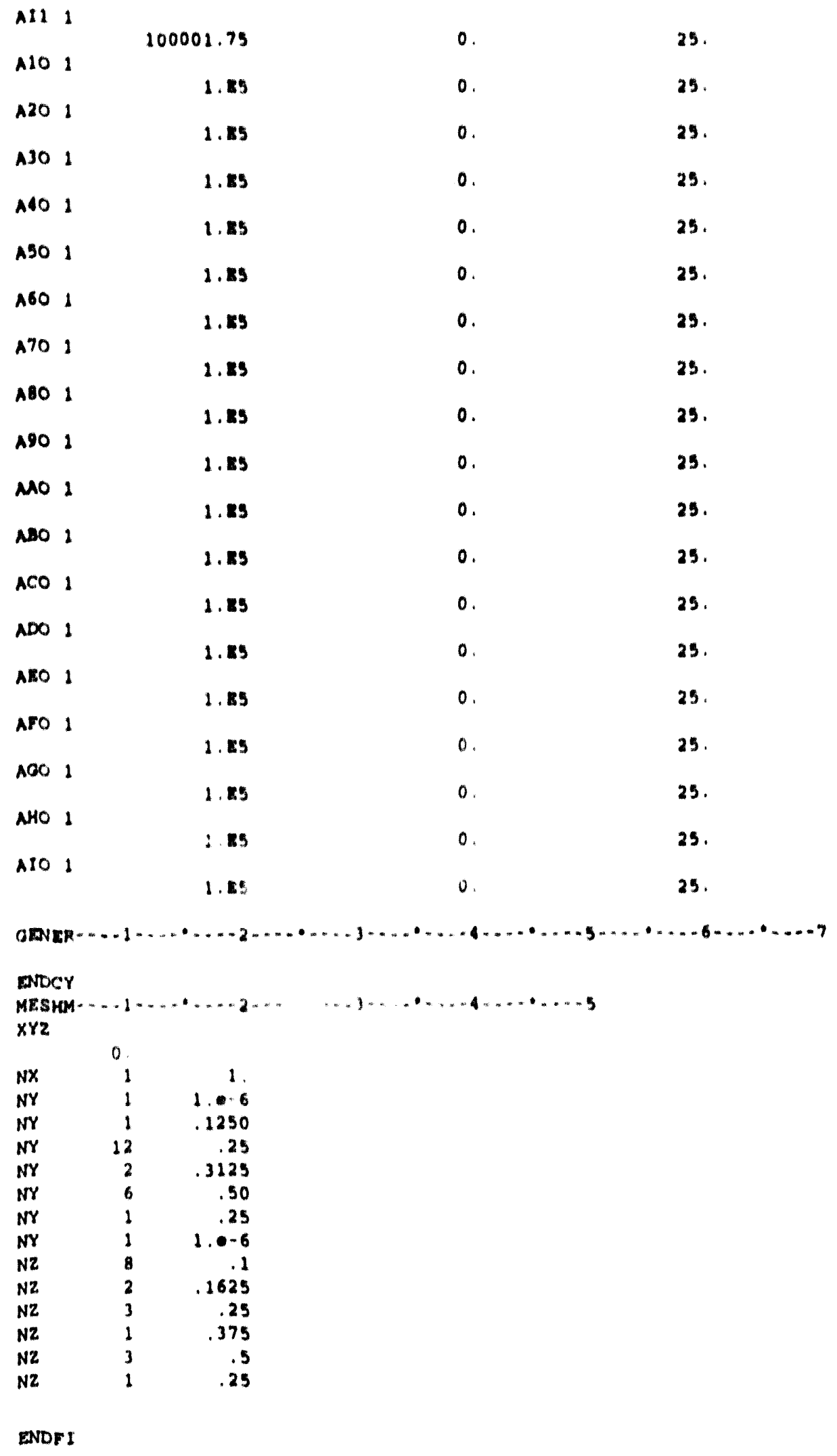

Fig. 15. Input file for the two-dimensional transport problem (continued). 
$1.08 \mathrm{e} 4$ seconds was selected to diminish time discretization errors which would arise if the time step size is allowed to get very large.

The MESH file was created with MESHMAKER and subsequently edited to make the left-and right-hand side columns have effectively infinite volume so that they may remain isobaric. The pressures at each side are set 10 produce a pore velocity of $0.1 \mathrm{~m} /$ day. The optional parameter MOP(13) was set equal to 0 so that the top of the domain is effectively a plane of symmetry. The flow domain boundary is defaulted to be the same as the calculation domain boundary.

Global results from the coarse grid for the tracer concentration at $t=20$ days are shown in the contour plot of Fig. 16. While Fig. 16 is qualitatively reasonable, it is difficult to rigorously compare these two-dimensional results with the analytical solution. For the purposes of rigorous comparison, one-dimensional cross-sections through the twodimensional fields were made and are shown compared to the same cross-sections from the analytical solution in Figs. 17-19. Fig. 17 shows the tracer profile result at $Z=-.15 \mathrm{~m}$ (line A-A' in Fig. 16) as calculated numerically with DISF for the coarse and fine grid along with the analytical result. Note that this profile is just below the top of the domain, and the source extends to $\mathrm{Z}=. .5 \mathrm{~m}$. Just as in the one-dimensional problem, numerical dispersion occurs in the numerical result. Fig. 18 shows the tracer profile at $Z=-.75 \mathrm{~m}$ (B-B' in Fig. 16), just below the line source which extends from $Z=0 \mathrm{~m}$ to $Z=-.5 \mathrm{~m}$. This profile shows the longitudinal dispersion of tracer which has dispersed transversally. Note the scale of the $\mathrm{C} / \mathrm{C}_{0}$ axis in Fig. 18. Shown in Fig. 19 is a profile at $\mathrm{Y}=2 \mathrm{~m}\left(\mathrm{C}-\mathrm{C}^{\prime}\right.$ in Fig. 16). At $t=20$ days, the front has moved approximately $2 \mathrm{~m}$ in the $\mathrm{Y}$-direction. Thus, the $C-C^{\prime}$ profile measures the transverse dispersion which occurs. Good agreement with the analytical result is seen. 


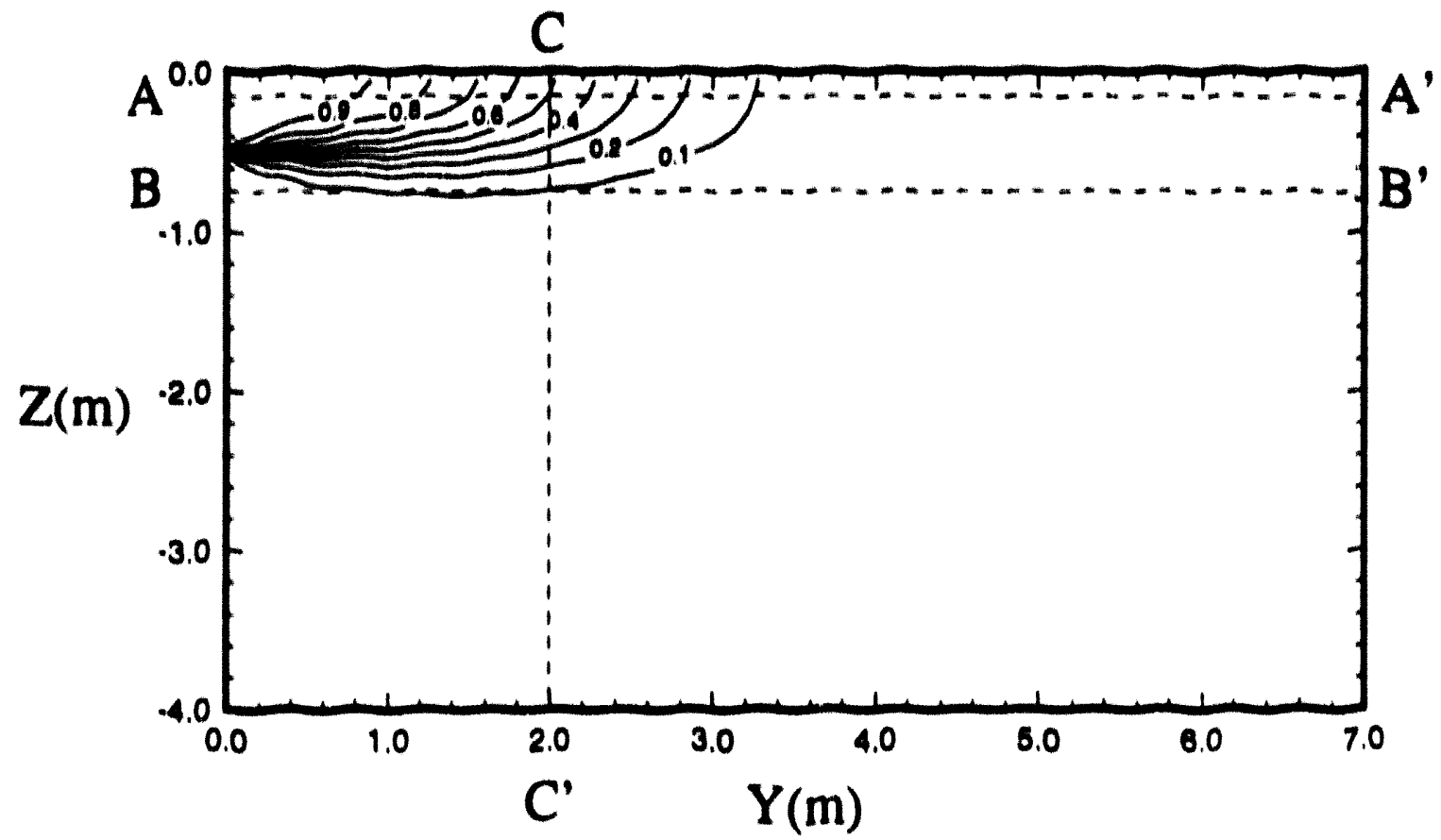

Fig. 16. Contour plot of tracer concentration at $t=20$ days along with the crosssections of Figs. 17-19. 


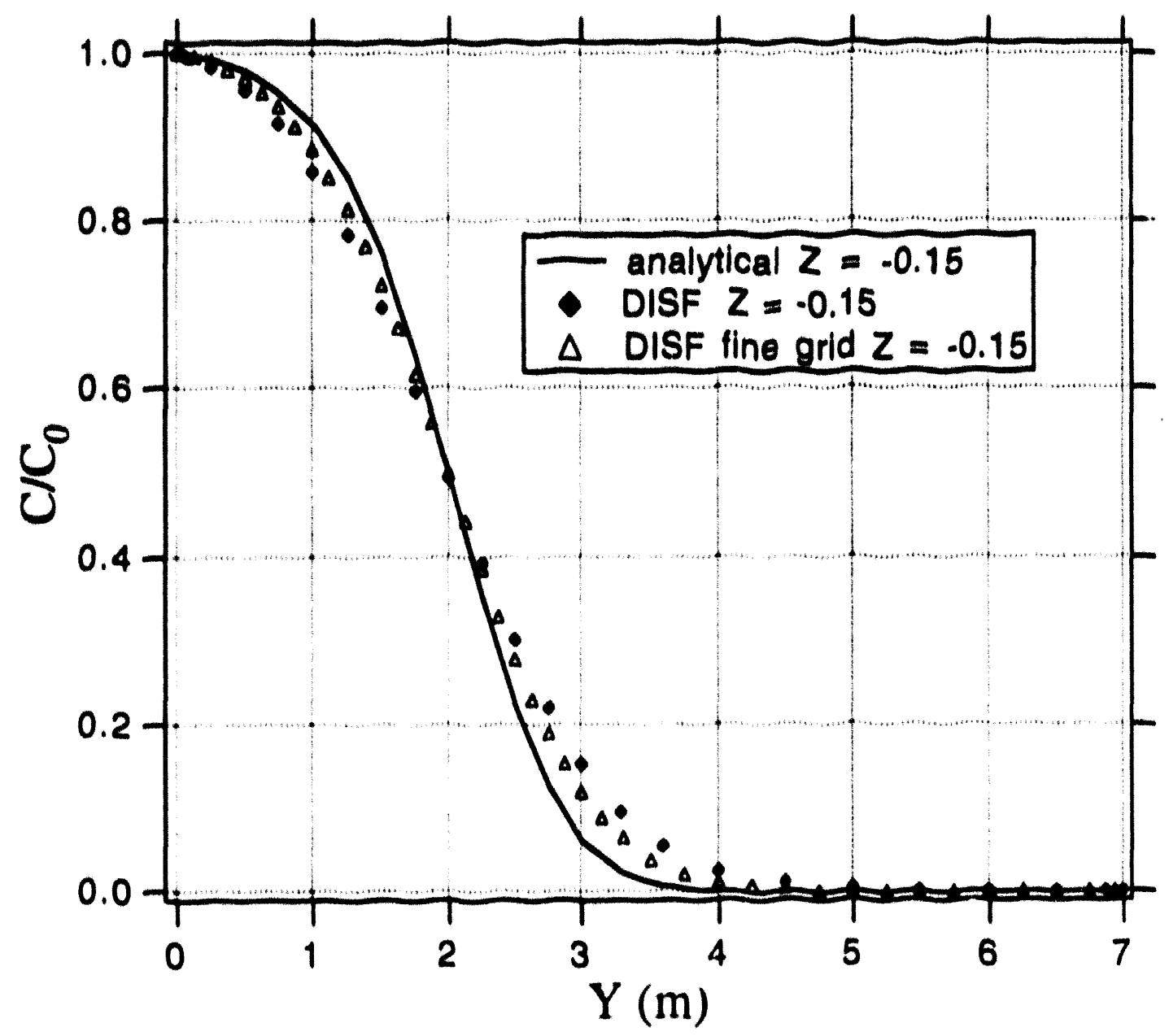

Fig. 17. Tracer brine concentration profile $A-A^{\prime}(Z=-.15 \mathrm{~m})$ for analytical and numerical calculations at $\ell=20$ days. 


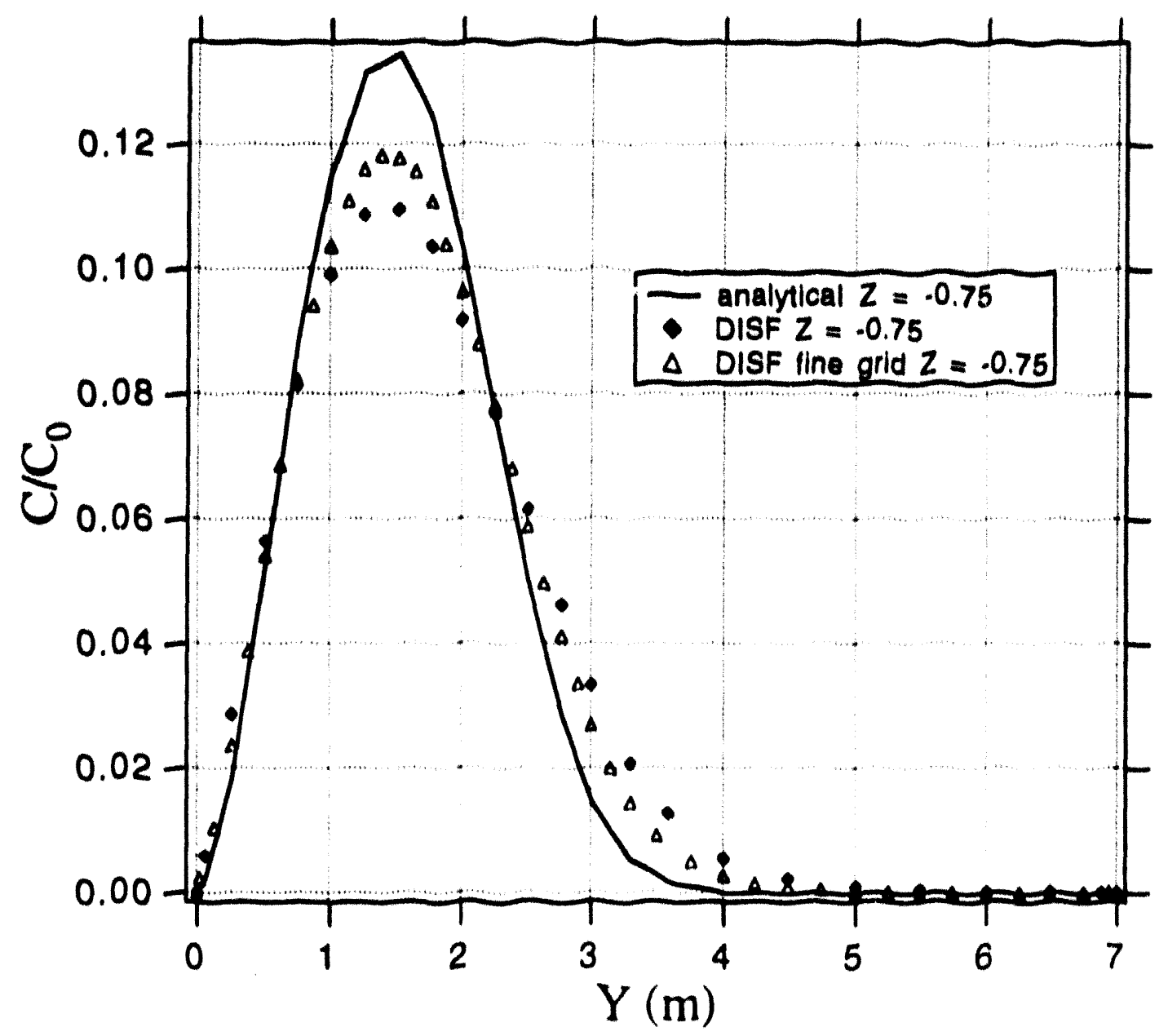

Fig. 18. Tracer brine concentration profile $B-B^{\prime}(Z=-.75 \mathrm{~m})$ for analytical and numerical calculations at $t=20$ days. 


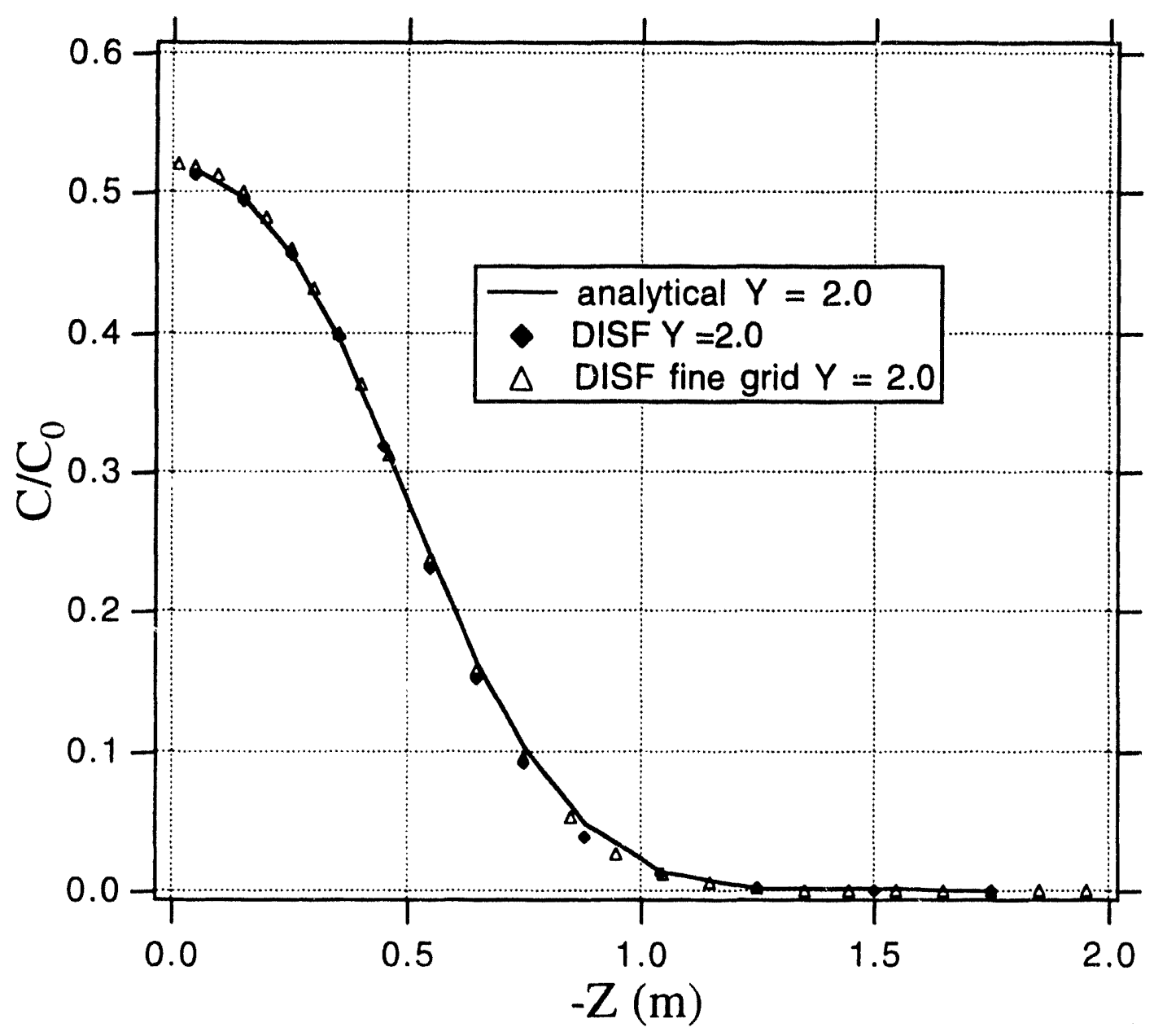

Fig. 19. Tracer brine concentration profile $\mathrm{C}^{-\mathrm{C}^{\prime}}(\mathrm{Y}=2 \mathrm{~m})$ for analytical and numerical calculations at $t=20$ days. 


\subsection{Two-Dimensional Brine Transport with Variable Density}

In order to demonstrate further capabilities of T2DM along with an example of the practical procedures used in T2DM for setting up a typical dispersion problem, we present here the results of a problem of two-dimensional hydrodynamic dispersion with variable density. This example problem closely resembles problem 1.5 of the Hydrocoin code verification study (Andersson et al., 1986; The International Hydrocoin Project, Level 3, 1992) and considers the saturated flow of groundwater over a salt dome including the effects of variable density due to brine concentration. In the problem, fresh water enters from the top boundary on the left-hand side, flows past a source of dense brine at the bottom, and then flows out through the top boundary on the right-hand side (Fig. 20). In the original problem specification (Andersson et al., 1986), the brine source is situated at the bottom along a boundary where the velocity is specified as being zero. In the absence of molecular diffusion, hydrodynamic dispersion woulis not be capable of picking up any of this brine if the flow velocity were identically zerc at the boundary where the brine source is located. In this example, we will demonstrate the use of the flow domain boundary option to model this bottom boundary condition.

The model domain and discretization are shown in Fig. 20 along with the top pressure boundary condition that provides the driving force for the flow. The grid has $30 \mathrm{x}$ $20=600$ blocks with $\Delta Y=30 \mathrm{~m}$, and $\Delta \mathrm{Z}=15 \mathrm{~m}$. For the purpose of representing boundary conditions, the grid has two additional rows of blocks, one at the top and one at the bottom, that are too thin $(\Delta Z=1 . e-6 \mathrm{~m})$ to appear in Fig. 20. Grid generation with the MESHMAKER facility is made prior to the simulation, and the grid is edited "by hand" to implement the desired boundary conditions. The thin grid blocks along the top are assigned very large volumes ( $>1 . e 40 \mathrm{~m}^{3}$ ) and therefore their initial conditions remain time-invariant, making these grid blocks effectively inactive. The inactive top row of grid blocks maintains the linear pressure profile of Fig. 20. Specification of arbitrary grid block 
(a)

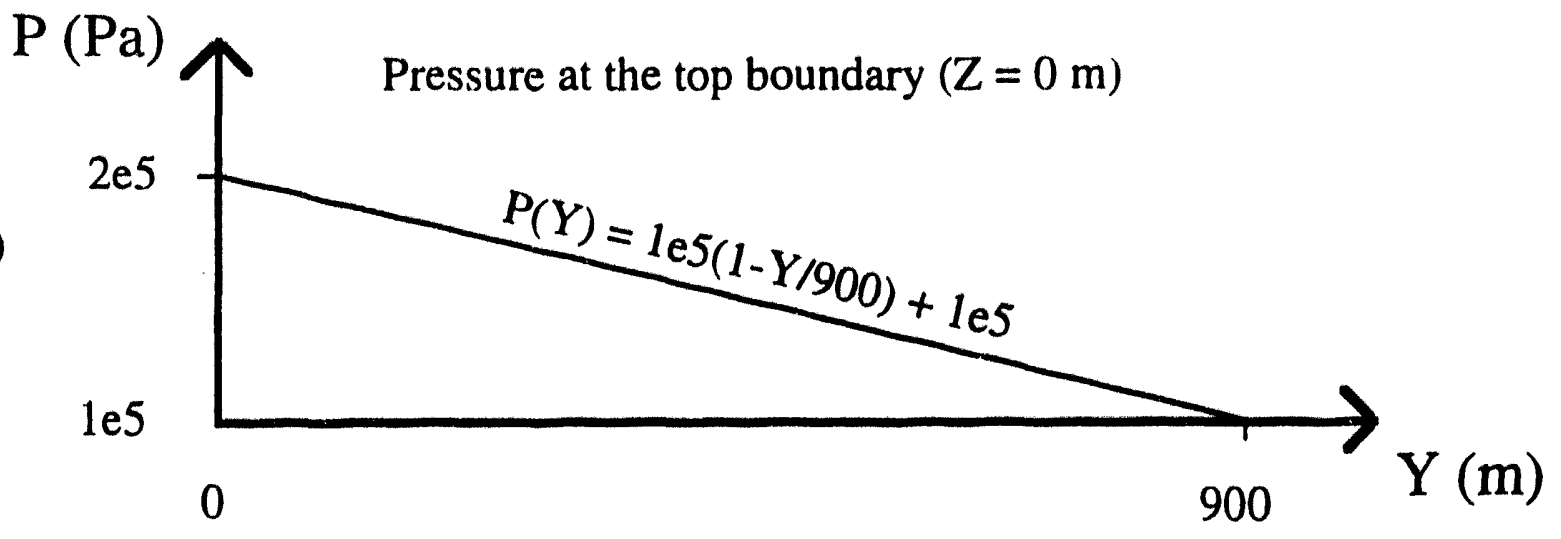

(b)

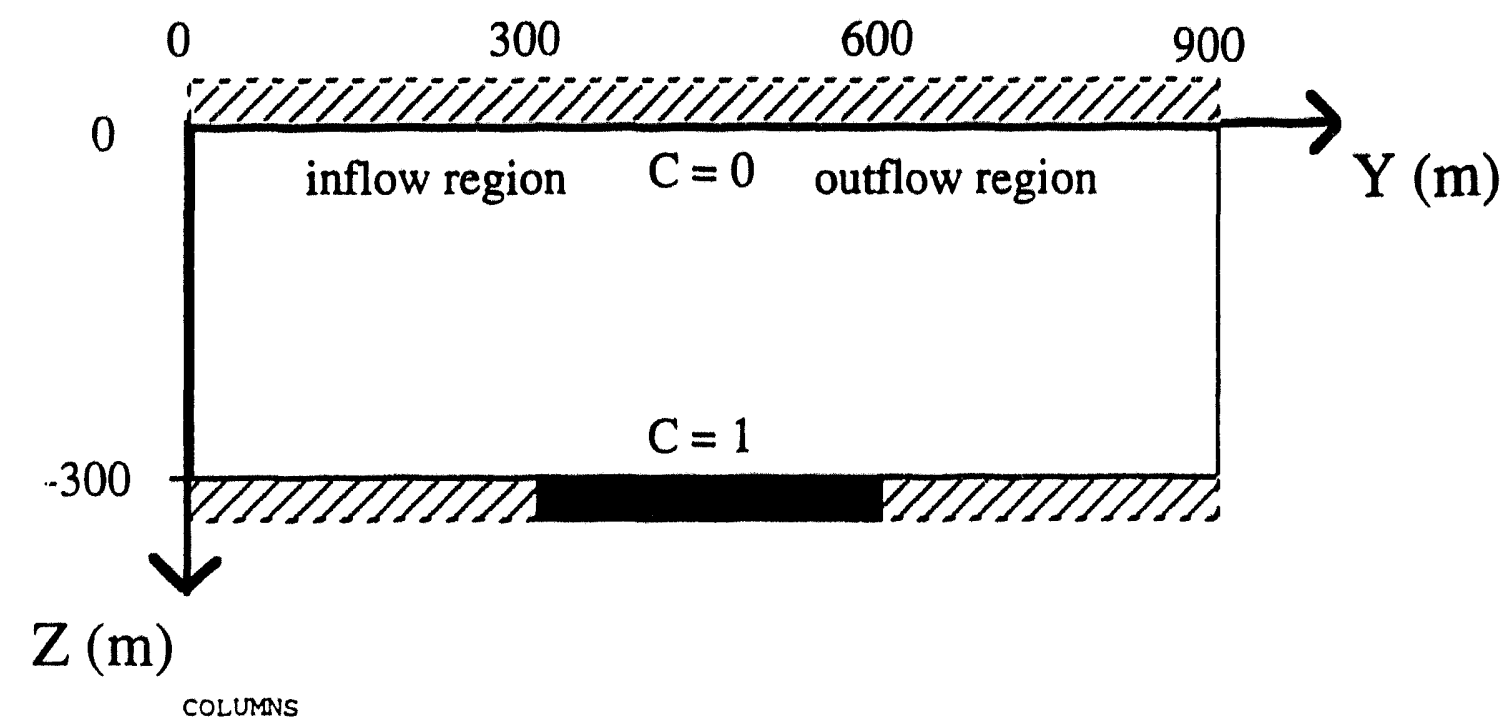

(c)

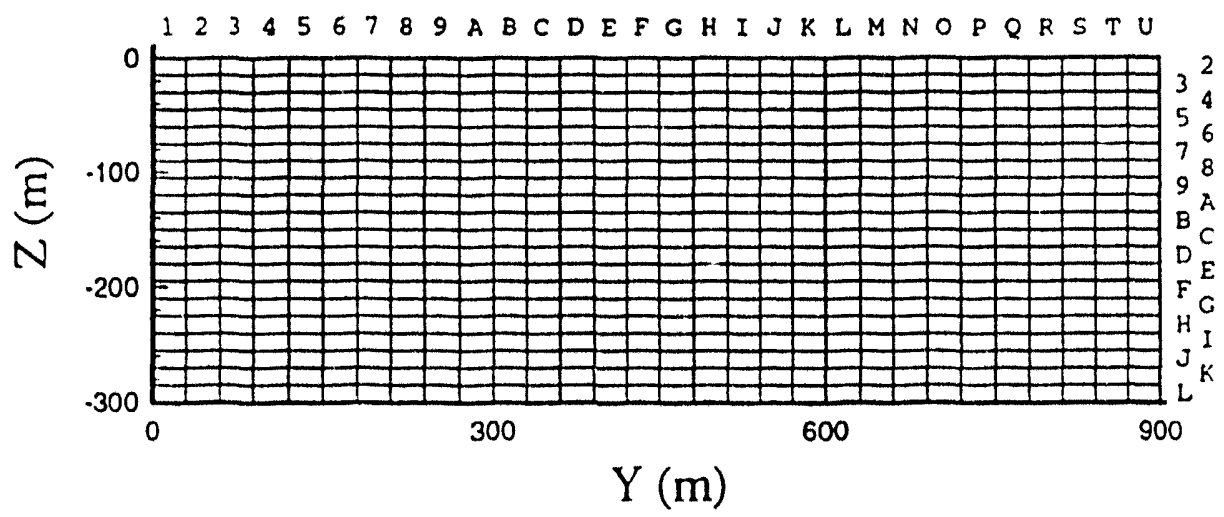

Fig. 20. Variable density brine flow and dispersion problem. (a) Top pressure boundary condition. (b) Schematic of flow domain, with top and bottom boundary blocks shown filled by diagonal lines. (c) Flow domain discretization of $30 \times 20$ blocks with $\Delta Y=30 \mathrm{~m}$ and $\Delta Z=15 \mathrm{~m}$. Grid blocks that are very small or outside the flow domain are not shown. 
volumes and interfacial areas allows the modeling of a brine source $(C=1)$ along one portion of the bottom row and a no flux $(d C / d Z=0)$ boundary condition in the remaining parts. Specifically, the grid blocks in the bottom row (below $Z=-300 \mathrm{~m}$ ) are given efiectively infinite volume in the region $300 \mathrm{~m}<\mathrm{Y}<600 \mathrm{~m}$ so that the initial concentration of the brine source $(C=1)$ will remain constant in those blocks. The interfacial areas of these grid blocks are unaltered in the CONNE block of the MESH file. The grid block volumes in the remaining parts of the bottom row retain their original volumes, but their interfacial areas for connections to grid blocks in the second-to-last row are set equal to zero in the CONNE block of the MESH file. This implements a "no-flow" boundary at the bottom for $0<\mathrm{Y}<300 \mathrm{~m}$ and $600<\mathrm{Y}<900 \mathrm{~m}$. The interface areas between the grid blocks in the last row at the boundary between the brine source and the regions $Y<300 \mathrm{~m}$ and $\mathrm{Y}>600 \mathrm{~m}$ are also set to zero in the CONNE block to prevent brine from leaving the brine source in the horizontal $(\mathrm{Y})$ direction.

The input file for this example problem is shown in Fig. 21. The option MOP(13) $=3$ is used to specify nearest-neighbor values for velocities and concentration gradients at boundaries. The option NFBB $=21$ identifies the row of grid blocks from $Z=-285 \mathrm{~m}$ to Z $=-300 \mathrm{~m}$ as the last row of grid blocks within the flow domain. In this way, we can obtain finite velocities at the interface to the $C=1$ brine boundary, which is essential for creating a flux due to transversal dispersion across the boundary. The input for MESHMAKER is included in the input file. Changes to the mesh file as generated by MESHMAKER are made with a text editor as described above. The top row is rock type TOP, the bottom row is rock type BOTT and the rest of the domain is type FINE. The parameters used are approximately those specified in the Hydrocoin 1.5 problem (shown in Table 3), the exceptions being that realistic internally calculated thermophysical properties of water (viscosity and density) are used in TOUGH2 rather than the arbitrary approximations specified for the Hydrocoin 1.5 problem. Initial conditions were zero velocity and fresh water everywhere except in the brine source grid blocks which contain pure brine. 
Table 3. Parameters for the variable density dispersion problem.

\begin{tabular}{llll}
\hline symbol & quantity & value & units \\
\hline$\phi$ & porosity & .2 & - \\
$k$ & permeability & $1 . e-12$ & $\mathrm{~m}^{2}$ \\
$\mu$ & viscosity & $8.9 \mathrm{e}-4$ & $\mathrm{~Pa} \mathrm{~s}$ \\
$\rho$ & brine density & $1.2 \mathrm{e} 3$ & $\mathrm{~kg} \mathrm{~m}^{-3}$ \\
$\delta$ & gravity & 9.80665 & $\mathrm{~m} \mathrm{~s}^{-2}$ \\
$\alpha T$ & transverse dispersivity & 2. & $\mathrm{~m}$ \\
$\alpha$ & longitudinal dispersivity & 20. & $\mathrm{~m}$ \\
$d$ & molecular diffusivity & 0. & $\mathrm{~m}^{2} \mathrm{~s}^{-1}$ \\
\hline
\end{tabular}

By setting the viscosity coefficients equal to 1.e-19, we forced isoviscous conditions as specified in the Hydrocoin 1.5 problem. No specification of relative permeability and capillary pressure data is needed, as the flow system remains in single-phase liquid conditions throughout. Pressure initial conditions at the top boundary were generated in a separate simulation run involving only the grid blocks in domain TOP. For this, standard TOUGH2 was used with two inactive grid blocks with zero nodal distance attached to the top row on each end (to blocks $\mathrm{A} 111$ and $\mathrm{AlU}$ 1), with pressures of 2.e5 $\mathrm{Pa}$ and 1.e5 $\mathrm{Pa}$. Pressures in this one-dimensional row were then run to steady state, and the resulting conditions were transferred from disk file SAVE to the INCON data block.

Steady-state results for the problem are shown in Fig. 22. The calculated results show that fresh water picks up saline brine from the bottom brine boundary by hydrodynamic dispersion. Advection and dispersion then transport the plume of salty water generally from left to right. Our results have some similarities but also important differences to previous work by Herbert et al. (1988). Further discussion is presented in Oldenburg and Pruess (1993). 


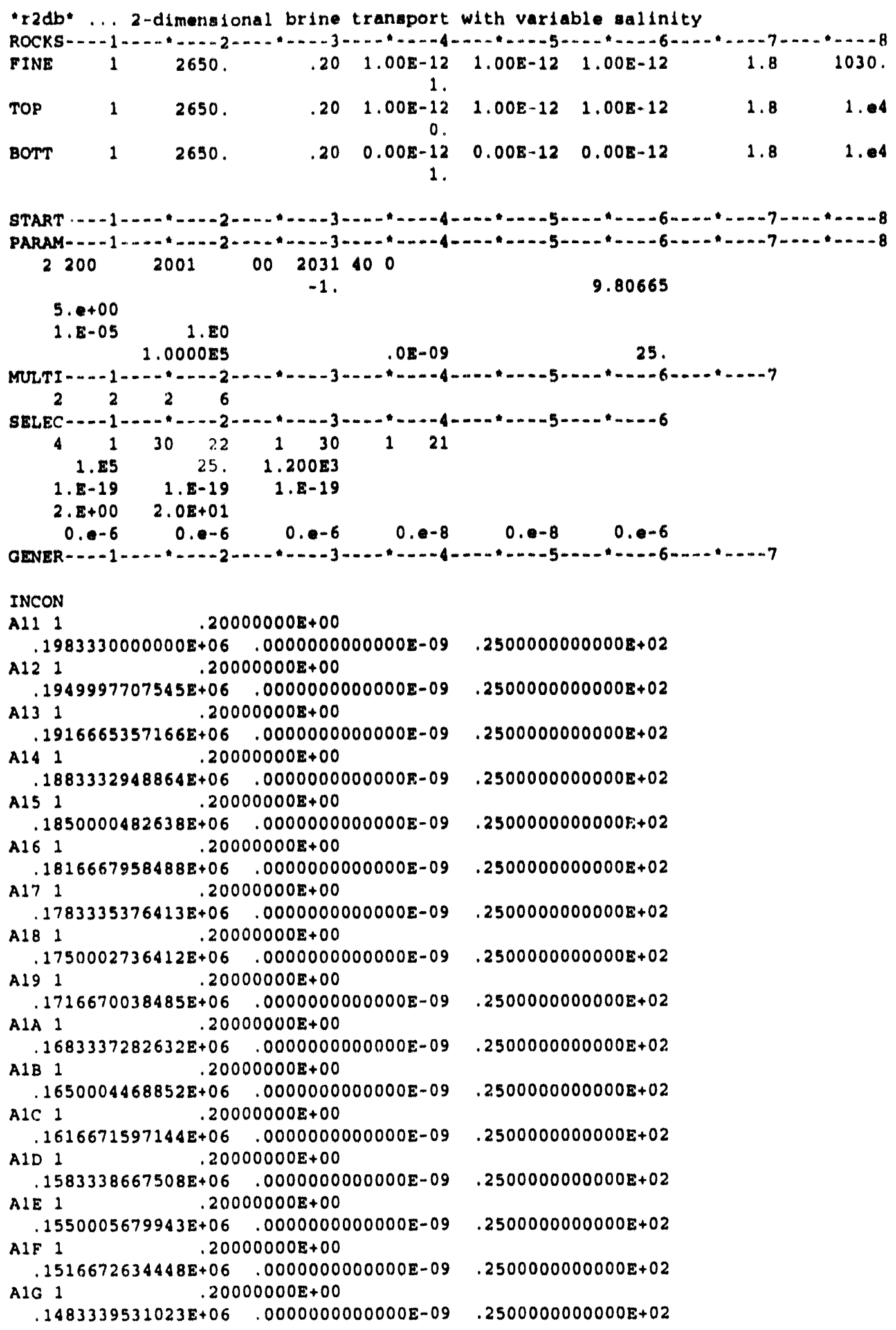

Fig. 21. Input file for the variable density dispersion problem. 


\begin{tabular}{|c|c|c|}
\hline AIH 1 & $00000 E+00$ & \\
\hline $.1450006369667 E+06$ & $.0000000000000 E-09$ & $2500000000000 \mathrm{E}+02$ \\
\hline A I 1 & $00000 \mathrm{E}+00$ & \\
\hline $1416673150380 E+06$ & $.0000000000000 \mathrm{E}-09$ & $.2500000000000 E+02$ \\
\hline AlJ 1 & $00000 E+00$ & \\
\hline $.1383339873160 E+06$ & .0000000000000 E-09 & $.2500000000000 E+02$ \\
\hline A1K 1 & $00000 E+00$ & \\
\hline $.1350006538008 E+06$ & $.0000000000000 \mathrm{E}-09$ & $.2500000000000 E+02$ \\
\hline ALL 1 & $00000 \mathrm{E}+00$ & \\
\hline $1316673144921 E+06$ & $.0000000000000 E-09$ & $.2500000000000 \mathrm{E}+02$ \\
\hline A1M 1 & $00000 E+00$ & \\
\hline $.1283339693900 \mathrm{E}+06$ & $.0000000000000 \mathrm{E}-09$ & $.2500000000000 \mathrm{E}+02$ \\
\hline$A 1 N 1$ & $00000 \mathrm{E}+00$ & \\
\hline $.1250006184944 E+06$ & $.0000000000000 \mathrm{E}-09$ & $.2500000000000 \mathrm{E}+02$ \\
\hline A10 1 & $00000 E+00$ & \\
\hline $.1216672618052 E+06$ & $.0000000000000 \mathrm{E}-09$ & $.2500000000000 \mathrm{E}+02$ \\
\hline ALP 1 & $00000 E+00$ & \\
\hline $.1183338993223 E+06$ & $.00000000000008 \times 09$ & $.2500000000000 \mathrm{E}+02$ \\
\hline A10 1 & $00000 E+00$ & \\
\hline $.1150005310457 E+06$ & $.0000000000000 \mathrm{E}-09$ & $.2500000000000 E+.72$ \\
\hline ALR 1 & $00000 \varepsilon+00$ & \\
\hline $.1116671569752 \mathrm{E}+06$ & $.0000000000000 \mathrm{E}-09$ & $.2500000000000 E+02$ \\
\hline ALs 1 & $00000 E+00$ & \\
\hline $.1083337771108 E+06$ & $.0000000000000 \mathrm{E}-09$ & $.2500000000000 z+C 2$ \\
\hline A1T 1 & $00000 \mathrm{E}+00$ & \\
\hline $.1050003914524 \mathrm{E}+06$ & $.0000000000000 E \cdot 09$ & $.2500000000000 \mathrm{E}+02$ \\
\hline A:O 1 & $00000 E+00$ & \\
\hline $.1016670000000 E+06$ & .0000000000000 E-09 & $.2500000000000 \mathrm{E}+02$ \\
\hline AM1 1 & $00000 \mathrm{E}+00$ & \\
\hline $.3000000000000 \mathrm{E}+07$ & $.0000000000000 \mathrm{E}=09$ & $.2500000000000 E+02$ \\
\hline AM2 1 & $00000 E+00$ & \\
\hline $.3000000000000 \mathrm{E}+07$ & $.0000000000000 E-09$ & $.2500000000000 \mathrm{E}+02$ \\
\hline AM3 1 & $00000 E+00$ & \\
\hline $.3000000000000 E+07$ & $.0000000000000 E-09$ & $.2500000000000 \mathrm{E}+02$ \\
\hline AM4 1 & $00000 E+00$ & \\
\hline $.3000000000000 \mathrm{E}+07$ & $.0000000000000 E-09$ & $.2500000000000 E+02$ \\
\hline AM5 1 & $00000 E+00$ & \\
\hline $.3000000000000 E+07$ & $.0000000000000 E-09$ & $.2500000000000 E+02$ \\
\hline AM6 1 & $00000 E+00$ & \\
\hline $.3000000000000 E+07$ & $.0000000000000 \mathrm{E}-09$ & $.2500000000000 \mathrm{E}+02$ \\
\hline AM7 1 & $00000 E+00$ & \\
\hline $.3000000000000 \mathrm{E}+07$ & $.0000000000000 E-09$ & $.2500000000000 E+02$ \\
\hline AM8 1 & $00000 E+00$ & \\
\hline $.30000000000008+07$ & $.0000000000000 \mathrm{E}-09$ & $.2500000000000 E+02$ \\
\hline AM9 1 & $00000 E+00$ & \\
\hline $.3000000000000 E+07$ & $.0000000000000 E-09$ & $.2500000000000 E+02$ \\
\hline AMA 1 & $00000 E+00$ & \\
\hline $.3000000000000 E+07$ & $.0000000000000 E-09$ & $.2500000000000 E+02$ \\
\hline AMB 1 & $00000 E+00$ & \\
\hline $.3000000000000 E+07$ & $1000000000000 \mathrm{E}+01$ & $2500000000000 \mathrm{E}+02$ \\
\hline AMC 1 & $00000 E+00$ & \\
\hline $.3000000000000 E+07$ & $.1000000000000 E+01$ & $.2500000000000 E+02$ \\
\hline AND 1 & $00000 E+00$ & \\
\hline $.3000000000000 \mathrm{E}+07$ & $.1000000000000 \mathrm{E}+01$ & $.2500000000000 E+02$ \\
\hline AME 1 & $00000 E+00$ & \\
\hline $.3000000000000 E+07$ & $.1000000000000 \varepsilon+01$ & $.2500000000000 E+02$ \\
\hline AMF 1 & $00000 E+00$ & \\
\hline $.3000000000000 E+07$ & $.1000000000000 \mathrm{E}+01$ & $.2500000000000 E+02$ \\
\hline AMG 1 & $00000 E+00$ & \\
\hline $.3000000000000 E+07$ & $.1000000000000 E+01$ & $.2500000000000 E+02$ \\
\hline
\end{tabular}

Fig. 21. Input file for the variable density dispersion problem (continued). 


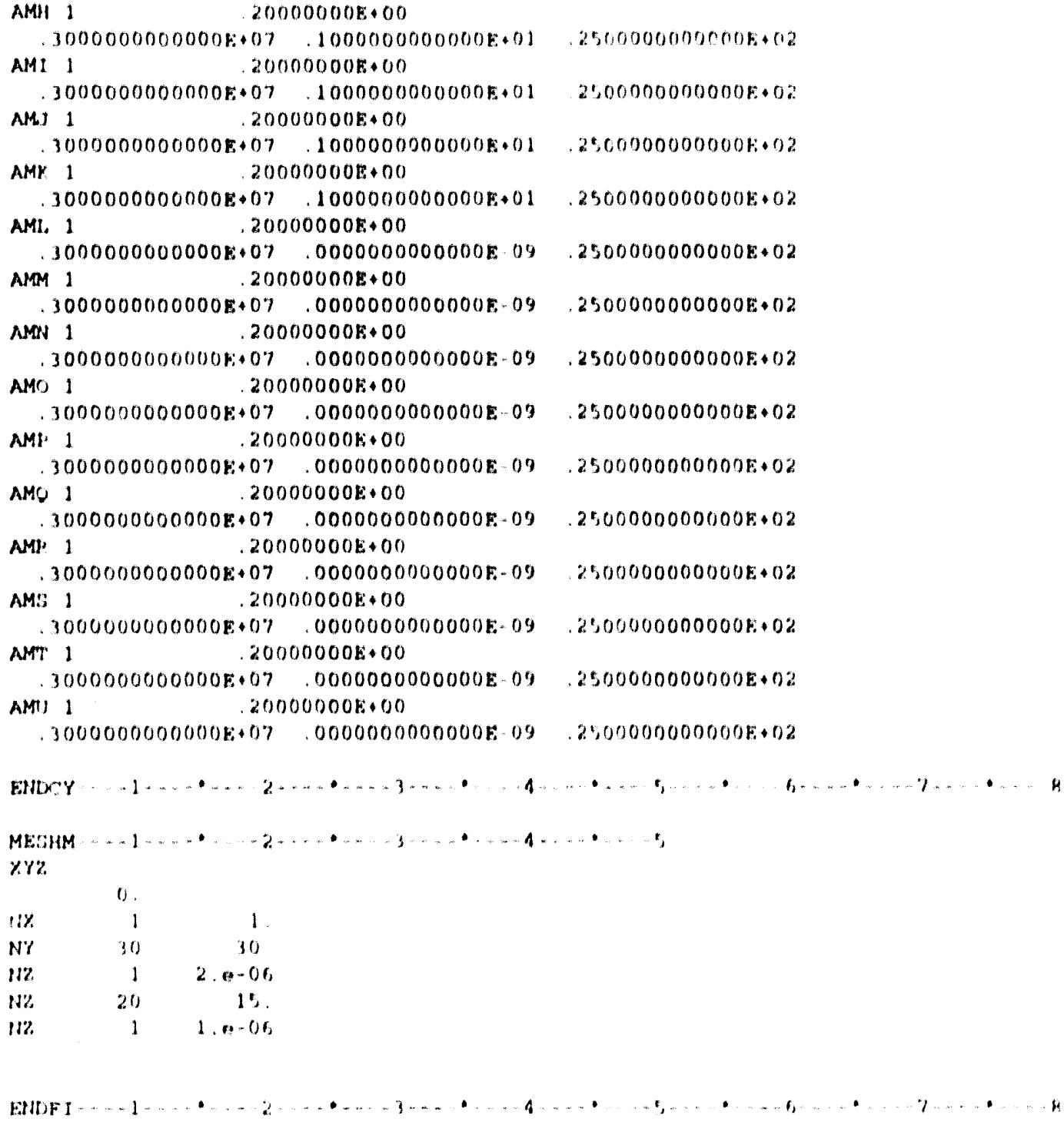

Fig. 21. Input file for the variable density dispersion problem (continued). 


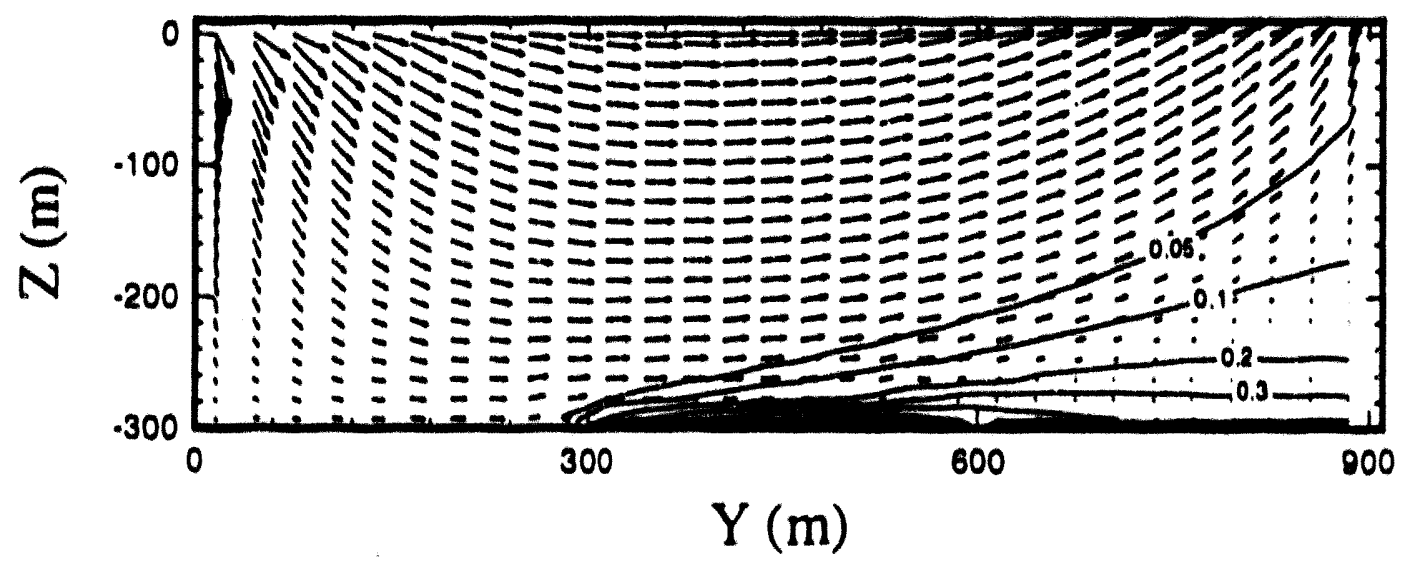

Fig. 22. Results for the variable density dispersion problem. Plot shows vectors of Darcy velocity and contours of brine mass fraction. 


\section{Final Notes}

This final section serves as a reiteration of important information necessary to use T2DM, the dispersion module for TOUGH2.

- The dispersion module T2DM requires that the MESH file represent a twodimensional Y-Z grid, have grid blocks in their correct physical positions, be ordered by-columns, and be at least 2 grid blocks on each side ( $Y$ and Z). The MESH must have grid-block-centered nodes.

- The user must input the number of grid blocks in each direction of the MESH. This is done in the SELEC block of the TOUGH2 input file. The number of grid blocks in the X-direction must always be 1 .

- The Iwo-dimensional section modeled is always a $Y \cdot Z$ section; users will set the gravitational acceleration equal to zero to model the horizontal plane.

- In order to model molecular diffusion effects, the tortuosity factor for the material must be included in the ROCK block in the input file. If not specified, the tortuosity will default to zero and no molecular diffusion will occur regardless of the molecular diffusivity entered in the SELEC block.

- The option MOP(8) controls diagnostic printout from DISF, with increasing values producing larger and more detailed printout from DISF. Normally. $\operatorname{MOP}(8)$ will be defaulted to zero.

- MOP(13) selects several options for the composition gradient vector and the Darcy velocity vector at the flow domain boundary. Typical problems will use $\operatorname{MOP}(13)=3$, for composition gradient and Darcy velocity vector to be nearest neighbor. This option affects only interpolated vector components.

- $\operatorname{MOP}(23)$ handles the treatment of non-direct terms in the Jacobian matrix. If $\operatorname{MOP}(23)=1$, the influence of neighbor grid blocks is ignored and the Jacobian matrix will be slightly less accurate although significantly sparser 
(fewer non-zero values), making the linear equation solution faster. If $\operatorname{MOP}(23)=0$ (the default), the full Jacobian is used. Using the full Jacobian will allow larger time steps, especially in strongly-coupled flow problems.

- In DISF, array dimensioning for the maximum number of interfaces (connections), grid blocks in the Y-direction, and grid blocks in the Zdirection is made with the parameters MNI, MNYY, and MNZZ, respectively. These parameters can be changed in the DISF subroutine by editing the parameter statement near the top of the DISF subroutine, as well as in the DOT subroutine for parameter MNI. The values selected for the dimensioning parameters are printed on output on the page with header "Summary of Program Units Used."

\section{References}

Andersson, K., B. Grundfelt, D.P. Hodgkinson, B. Lindbom, and C.P. Jackson, HYDROCOIN Level / Final Report: Verification of Groundwater Flow Models, Swedish Nuclear Power Inspectorate (SKi), 1986.

deMarsily, Ghislain, Quantitative Hydrogeology, Academic Press, pp. 230-247, 1986.

Javandel, Iraj, Christine Doughty, and Chin-Fu Tsang. Groundwater Transport, Handbook of Mathematical Models, American Geophys. Union, pp. 14-19, 1984.

Herbert, A.W., C.P. Jackson, and D.A. Lever, Coupled groundwater flow and solute transport with fluid density strongly dependent upon concentration. Water Resources Research, vol. 24, no. 10, pp. 1781-1795, 1988. 
The Intermational Hydrocoin Project, Level 3: Uncertainty and Sensitivity Analysis, Swedish Nuclear Power Inspectorate (SKi), Nuclear Energy Agency (NEA), 1992.

Oldenburg, C.M. and K. Pruess, Dispersive transpon dynamics in a strongly coupled groundwater-brine flow system, submitted to Water Resources Research, 1993.

Pruess, K., TOUGH User's Guide, Nuclear Regulatory Commission, Report NUREG/CR-4645, June 1987 (also Lawrence Berkeley Laboratory Report LBL-20700, Berkeley, California, June 1987).

Pruess, K., TOUGH2 - A General Purpose Numerical Simulator for Multiphase Fluid and Heat Flow, Lawrence Berkeley Laboratory Repon LBL-29400, Berkeley, California, May 1991).

Pruess, Karsten, EOS7, An equation-of-state module for the TOUGH2 simulator for twophase flow of saline water and air, Lawrence Berkeley Laboratory Report LBL.31114. Berkeley, California. August 1991.

Sahimi, M., B.D. Hughes, L.E. Scriven, and H.T. Davis, Dispersion in flow through porous media-1. One-phase flow, Chem. Eng. Sci., 4I(8), 2103-2122, 1986.

Sahimi, M., B.D. Hughes, L.E. Scriven, and H.T. Davis, Dispersion in flow through porous media-11. Two-phase flow, Chem. Eng. Sci, 4/(8), 2123-2136, 1986. 


\section{Acknowledgments}

This work was supported by the WIPP project, Sandia National Laboratories, under Document Number 129847, and by the Assistant Secretary for Energy Efficiency and Renewable Energy, Geothermal Division, U.S. Department of Energy, through contract No. DE-AC03-76SF00098. The authors acknowledge helpful discussions with Steve Webb throughout the course of this work. Reviews of a preliminary version of this report by Stefan Finsterle and George Moridis are greatly appreciated. We also thank Mohsen Alavi and George Moridis for helpful discussions on the numerical modeling of dispersion processes.

\section{Nomenclature}

length of line source

m

$d$

molecular diffusivity

$m^{2} s^{-1}$

C

concentration

$\mathrm{kg} \mathrm{m-3}$

D

connection distance

m

D

dispersion coefficient

$\mathrm{m}^{2} \mathrm{~s}^{-1}$

$\overline{\mathbf{D}}$

dispersion tensor

$m^{2} s^{*}=1$

c

grid block to the east

E.

interface to the east

$\mathbf{8}$

acceleration of gravity vector

$m s^{-2}$

$\mathbf{F}$

Darcy flux vector

$k g m^{2} s^{-1}$

j

diffusive flux

$k g m^{2} s^{-1}$

$k$

permeability

$\mathrm{m}^{2}$

$k_{r}$

relative permeability

$\boldsymbol{K}$

effective conductivity term $\phi S \tau$.

M

mass accumulation term

$\mathrm{kg} \mathrm{m}^{-3}$

MNCON Maximum number of connections (interfaces) 
n

n

N

NEQ

NK number of mass components (species)

NPH maximum number of phases present

\begin{tabular}{|c|c|c|}
\hline$P$ & pressure & $\mathrm{Pa}$ \\
\hline 9 & source term & $\mathrm{kg} \mathrm{m}^{-3} \mathrm{~s}^{-1}$ \\
\hline $\mathbf{s}$ & grid block to the south & \\
\hline $\mathbf{S}$ & interface to the south & \\
\hline$S$ & phase saturation & \\
\hline$t$ & time & days \\
\hline v & coefficients of viscosity (see EOS7 report) & \\
\hline u & magnitude of the Darcy velocity vector & $\mathrm{m} \mathrm{s}^{-1}$ \\
\hline $\mathbf{u}$ & Darcy velocity vector & $\mathrm{ms}^{-1}$ \\
\hline$V$ & volume & $m^{3}$ \\
\hline U & Y-component of Darcy velocity & $\mathrm{ms}^{-1}$ \\
\hline$w$ & grid block to the west & \\
\hline W & interface to the west & \\
\hline$X$ & mass fraction & \\
\hline$Y$ & $Y$-coordinate & \\
\hline Z & Z.-coordinate & \\
\hline
\end{tabular}

Greek symbols

$\begin{array}{lll}\alpha & \text { intrinsic dispersivity } & \mathrm{m} \\ \Gamma & \text { surface area } & \mathrm{m}^{2} \\ \mu & \text { dynanic viscosity } & \mathrm{kg} \mathrm{m}^{-1} \mathrm{~s}^{-1} \\ \phi & \text { porosity } & \end{array}$

grid block to the north

outward unit normal vector

interface to the north

number of equations per grid block

pressure

$\mathrm{kg} \mathrm{m}^{* 3} \mathrm{~s}^{-1}$

$\mathrm{m}^{3}$

$\mathrm{m} \mathrm{s}^{-1}$ 